\title{
Flows on networks: recent results and perspectives
}

\author{
Alberto Bressan, Sunčica Čanić, Mauro Garavello, \\ Michael Herty, and Benedetto Piccoli ${ }^{3}$
}

The broad research thematic of flows on networks was addressed in recent years by many researchers, in the area of applied mathematics, with new models based on partial differential equations. The latter brought a significant innovation in a field previously dominated by more classical techniques from discrete mathematics or methods based on ordinary differential equations. In particular, a number of results, mainly dealing with vehicular traffic, supply chains and data networks, were collected in two monographs: Traffic flow on networks, AIMSciences, Springfield, 2006, and Modeling, simulation, and optimization of supply chains, SIAM, Philadelphia, 2010. The field continues to flourish and a considerable number of papers devoted to the subject is published every year, also because of the wide and increasing range of applications: from blood flow to air traffic management. The aim of the present survey paper is to provide a view on a large number of themes, results and applications related to this broad research direction. The authors cover different expertise (modeling, analysis, numeric, optimization and other) so to provide an overview as extensive as possible. The focus is mainly on developments which appeared subsequently to the publication of the aforementioned books.

\footnotetext{
${ }^{1}$ The author acknowledges partial support of 2013 GNAMPA project "Leggi di Conservazione: Teoria e Applicazioni”.

${ }^{2}$ The author acknowledges support by BMBF KinOpt, DFG Cluster of Excellence EXC128 and DAAD $54365630,55866082$.

${ }^{3}$ The author acknowledges partial support of NSF Research Network in the Mathematical Sciences KI-Net "Kinetic description of emerging challenges in multiscale problems of natural sciences" Grant \#: 1107444.

A. Bressan, Department of Mathematics, Penn State University, University Park, Pennsylvania, U.S.A.

E-mail: bressan@math.psu.edu

S. Čanić, Department of Mathematics, University of Houston, Houston, Texas, U.S.A.

E-mail: canic@math.uh.edu

M. Garavello, Department of Mathematics and Applications, University of Milano Bicocca, Milano, Italy

E-mail: mauro.garavello@unimib.it

M. Herty, Center for Computational Engineering Science (CCES), RWTH Aachen University, Aachen, Germany

E-mail: herty@igpm.rwth-aachen.de

B. Piccoli, Department of Mathematical Sciences and Center for Computational and Integrative Biology, Rutgers University, Camden, New Jersey, U.S.A.

E-mail: piccoli@camden.rutgers.edu
} 
Mathematics Subject Classification (2010). 35L65, 35R02, 34H05.

Keywords. Networks, balance laws, control problems.

\section{Introduction}

The expression "flows on networks" is used to denote at the same time a collection of practical problems, ranging from vehicular traffic on urban roads to blood circulatory system in humans, as well as a wealth of mathematical models to represent and study such problems. Our point of view is that of macroscopic models, which describe the reality looking at aggregate quantities, such as average particle densities and velocities, and their evolution in time. More precisely, in the last ten to fifteen years, models based on partial differential equations proved to be useful for the analysis, simulation and control of network flows. In particular, for large systems they may present advantages over microscopic models, focusing on each particle of the system, and discrete time ones (such as discrete events). Obviously such advantages are more or less evident depending on the specific characteristics and dimensions of the given problem.

Let us start by providing some examples of engineering, bio-medical and socioeconomical networks. We will then turn to a specific mathematical framework, which will be the common theme for this review paper. In Figure 1, we represent in a compact way a collection of practical problems, which provides a reasonably rich selection of the possible applications of our methods. The common features are those of a state space described by a network (a topological graph) and a dynamics given by solutions to systems of partial differential equations. To start with, let us mention problems involving real fluids, thus physical flows on networks. These include irrigation channels with flowing water [51], [14], gas pipelines [10] and blood circulation [66]. The physics of these three problems is very different: in irrigation channels water presents one surface in contact with the air and the others in contact with the channel boundaries, while in gas pipelines there is a uniform (often circular) surface of contact with the tube. Finally, in blood flow, the boundary is as in gas pipelines, but the tube itself (vessel) is elastic and changes shape depending on the flow strength.

One of the most studied example is that of vehicular traffic, where fluid dynamic models were used, for a single road, since the 50s with the seminal work of LighthillWhitham and Richards (see [71] for the networks case). Another example is that of air traffic management, where the flow occurs on routes in the airspace [120].

Less expected applications include supply chains, where the first model was proposed in the seminal paper [6] passing to the limit in discrete-event queuing systems. Also data and telecommunication networks may be described by conservation laws, 


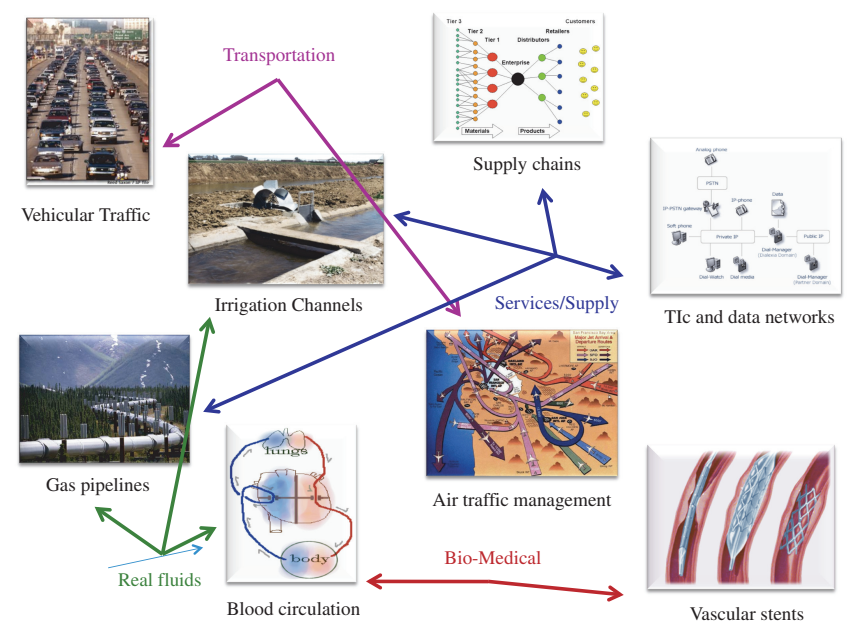

Figure 1. Applications of models based on partial differential equations.

under suitable assumptions, when looking the dynamics at intermediate time scales [58]. Finally, we will deal also with mechanical structures formed by elastic components, each admitting one dimensional descriptions. Our key example of that is a vascular stent placed in an artery [121], where the flow is linked to displacement waves.

Let us now pass to our general mathematical framework. Consider a system of balance laws in one space dimension of the form

$$
\partial_{t} u+\partial_{x} f(u)=g(t, x, u),
$$

where $t \in \mathbb{R}^{+}$is time, $x \in \mathbb{R}$ is the space variable, $f$ is the flow and $g$ the source term. More precisely, we are interested in the evolution equation (1) on a network (or topological graph), consisting of a collection of one-dimensional manifolds connected at nodes. In most cases (1) is equipped with further algebraic conditions imposed on vertices of the network. Fix a vertex $x_{*}$, then such conditions can be written compactly as

$$
\Psi\left(t, u\left(t, x_{*}+\right)\right)=0
$$

(assuming that each link adjacent to $x_{*}$ is parametrized by an interval of the type $\left.\left[x_{*}, b\right]\right)$. We will also consider the case of a time-varying vertex position, thus with $x_{*}=x_{*}(t)$. Then the coupling involves an ordinary differential equation for $x_{*}$ :

$$
\Psi\left(t, x_{*}(t), \dot{x}_{*}(t), \ddot{x}_{*}(t), u\left(t, x_{*}(t)+\right)\right)=0 .
$$

The physical properties of the system under consideration will induce a specific choice of the function $\Psi$. For instance, in vehicular traffic we will impose conservation of 
cars, traffic distribution coefficients and flow maximization. For simplicity, we focus on the case of a single node. This is not a major restriction, since, due to finite speed of propagation of waves, one can easily extend the results in the case of complex networks, see [71].

The paper is organized as follows. Section 2 presents results on existence of solutions and includes both the case of a single conservation law and systems of conservation laws. Section 3 provides various models to which the theory can be applied: vehicular traffic, gas pipelines, supply chains, data and telecommunication networks, irrigation channels and blood flow. Section 4 considers more complex situations where a coupling of different systems of equations (ODE-PDE or PDEPDE) is needed. Also in this case various examples are provided, such as the piston problem, sewer systems, supply chains and blood flow. Section 5 considers controlled systems, where the control acts within the coupling condition at the vertex $\Psi$. The main examples are provided by vehicular traffic and gas pipelines. Section 6 presents some numerical methods for conservation laws on networks. For sake of space we briefly review numerical coupling conditions for finite volume schemes and then show how numerical methods for multi-dimensional models can be used to deduce coupling conditions for one-dimensional reductions. Section 7 will present two specific applications of the developed methods: The study of optimal shape of vascular stents and vehicular traffic monitoring using GPS data from mobile sensors. Finally, Section 8 will present open problems and future perspectives of this rich research field.

\section{Theoretical results}

A general network is represented by a directed graph $\mathcal{E}$ composed by a finite number of edges connected by vertices or junctions. We use a directed graph to model the network, since for most of the applications we present here, there is a preferred direction for the network flow. For example, in an unidirectional car traffic road, vehicles direction is given a priori. The direction of particles may also be given by the presence of "pumps", compressors in gas pipeline network and the heart in the human circulatory system.

For the sake of simplicity, we restrict the attention to a very special network, composed by $n$ edges (labeled by $I_{i}$ and modeled by the real interval $(0, \infty)$ ) connected by a single junction $J$. We remark that this simplification does not imply a loss of generality: this is due to the fact that the hyperbolic systems we consider here, have the property that waves propagate with finite velocity. With similar arguments as in [71], one can prove that results for a single junction can be generalized to the case of an arbitrary network. 
Finally, we deal with the system

$$
\left\{\begin{aligned}
\partial_{t} u_{1}+\partial_{x} f_{1}\left(u_{1}\right) & =g_{1}\left(t, x, u_{1}\right) \\
& \vdots \\
\partial_{t} u_{n}+\partial_{x} f_{n}\left(u_{n}\right) & =g_{n}\left(t, x, u_{n}\right)
\end{aligned}\right.
$$

each equation being defined for $t \geq 0$ and $x \geq 0$, and coupled through the nodal condition

$$
\Psi\left(u_{1}(t, 0+), \ldots, u_{n}(t, 0+)\right)=0 .
$$

Here, for every $j \in\{1, \ldots, n\}, u_{j}:[0, T) \times I_{j} \rightarrow \Omega_{j}, f_{j}: \mathbb{R}^{N_{j}} \rightarrow \mathbb{R}^{N_{j}}, g_{j}:[0, T) \times$ $I_{j} \times \mathbb{R}^{N_{j}} \rightarrow \mathbb{R}^{N_{j}}, T \in(0,+\infty]$ and $\Omega_{j}$ is a subset of $\mathbb{R}^{N_{j}}$. In some applications, the function $\Psi$ defining the nodal condition (4) can depend explicitly also from time $t$; under suitable assumptions all the results of this section can be extended to this case. We supplement (3) and (4) with the initial condition

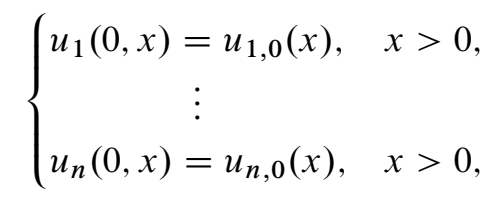

where, for every $j \in\{1, \ldots, n\}, u_{j, 0}: I_{j} \rightarrow \Omega_{j}$ are given functions. For brevity we introduce the notation

$$
u=\left[\begin{array}{c}
u_{1} \\
\vdots \\
u_{n}
\end{array}\right], \quad f(u)=\left[\begin{array}{c}
f_{1}\left(u_{1}\right) \\
\vdots \\
f_{n}\left(u_{n}\right)
\end{array}\right], \quad g(t, x, u)=\left[\begin{array}{c}
g_{1}\left(t, x, u_{1}\right) \\
\vdots \\
g_{n}\left(t, x, u_{n}\right)
\end{array}\right]
$$

and we rewrite (3), (4) and (5) in the form

$$
\left\{\begin{array}{l}
\partial_{t} u+\partial_{x} f(u)=g(t, x, u) \\
\Psi(u(t, 0+))=0 \\
u(0, x)=u_{0}
\end{array}\right.
$$

Definition 2.1. Fix $\hat{u}=\left(\hat{u}_{1}, \ldots, \hat{u}_{n}\right) \in \prod_{j=1}^{n} \Omega_{j}$ and $\left.\left.T \in\right] 0,+\infty\right]$. A function $u \in \mathcal{C}^{0}\left([0, T) ; \hat{u}+L^{1}\left(\mathbb{R}^{+} ; \prod_{j=1}^{n} \Omega_{j}\right)\right)$ is a weak solution to the Cauchy problem (7) on $[0, T)$ if the following conditions hold.

(1) For all $\varphi \in \ell_{c}^{\infty}(]-\infty, T\left[\times \mathbb{R}^{+} ; \mathbb{R}\right)$ and for $j \in\{1, \ldots, n\}$,

$$
\begin{aligned}
& \int_{0}^{T} \int_{\mathbb{R}^{+}}\left(u_{j} \partial_{t} \varphi+f_{j}\left(u_{j}\right) \partial_{x} \varphi\right) d x d t+\int_{\mathbb{R}^{+}} u_{j, 0}(x) \varphi(0, x) d x \\
& \quad=-\int_{0}^{T} \int_{\mathbb{R}^{+}} g \varphi d x d t
\end{aligned}
$$


(2) For a.e. $t \in(0, T)$, the limits $u_{j}(t, 0+)$ exist and satisfy the condition $\Psi\left(u_{1}(t, 0+), \ldots, u_{n}(t, 0+)\right)=0$.

In this section we present some existence results for the Cauchy problem (7). In general the steps for obtaining existence and well-posedness of such a problem are the following ones. First one considers the homogeneous case, i.e., $g=0$,

$$
\left\{\begin{array}{l}
\partial_{t} u+\partial_{x} f(u)=0 \\
\Psi(u(t, 0+))=0 \\
u(0, x)=u_{0}(x),
\end{array}\right.
$$

and applies the wave-front tracking technique (see for example [71]). This technique is based on the solutions to classical Riemann problems (see for example [23]) and to Riemann problems at the nodal points. More precisely, a Riemann problem at the node is the following Cauchy problem:

$$
\begin{cases}\partial_{t} u_{1}+\partial_{x} f_{1}\left(u_{1}\right)=0, & t>0, x \geq 0 \\ \quad \vdots & t>0, x \geq 0 \\ \partial_{t} u_{n}+\partial_{x} f_{n}\left(u_{n}\right)=0, & t>0, \\ \Psi\left(u_{1}(t, 0+), \ldots, u_{n}(t, 0+)\right)=0, & x \geq 0, \\ u_{1}(0, x)=\bar{u}_{1,0}, & x \geq 0 \\ \quad \vdots & \\ u_{n}(0, x)=\bar{u}_{n, 0}, & \end{cases}
$$

where, for every $j \in\{1, \ldots, n\}, \bar{u}_{j, 0} \in \Omega_{j}$.

Once well-posedness is obtained for $g=0$, the full case usually follows through operator splitting technique, along the lines initiated in [55]. The source term is treated as an ordinary differential equation, where $u$ depends on time $t$ and on the space variable as parameter. A Lipschitz type condition for $g$ and a uniform bound of $\left|\partial_{x} g(t, x, u)\right|$ by an $L^{1}$ function $K(x)$ are the classical assumptions, which guarantee the well-posedness of the system (7).

In general, solutions $u$ to the Cauchy problem (7) have BV regularity with respect to the space variable $x$. In the sequel, if $u$ is a BV function, then $\operatorname{TV}(u)$ denotes the total variation of the function $u$.

2.1. The scalar case. In this part we treat the scalar case, i.e. we assume that each equation in (3) is indeed a scalar equation, so that $N_{j}=1$ for every $j \in\{1, \ldots, n\}$. We also assume that the source terms $g_{i}$ are constantly equal to 0 .

Moreover we put $\Omega_{j}=[0,1]$ for every $j$ and on the fluxes $f_{j} \in \ell^{2}([0,1] ; \mathbb{R})$ we make the following assumptions: 
(1) $f_{j}(0)=f_{j}(1)=0$;

(2) either $f_{j}$ is strictly concave or $f_{j}$ is strictly convex.

Denote, for every $j \in\{1, \ldots, n\}$, with $\sigma_{j} \in(0,1)$ the unique point in $(0,1)$ such that $f_{j}^{\prime}\left(\sigma_{j}\right)=0$.

First, let us introduce the definition of the solution to the Riemann problem (9) in this case.

Definition 2.2. A collection of functions $u_{j} \in \mathcal{C}^{0}\left([0, T) ; L_{\mathrm{loc}}^{1}\left(I_{j}\right)\right)$ is a solution to the Riemann problem (9) if the following conditions hold.

(1) For every $j \in\{1, \ldots, n\}, k \in[0,1]$ and every $\tilde{\varphi}:\left[0, T\left[\times I_{j} \rightarrow \mathbb{R}\right.\right.$ smooth, positive with compact support in $] 0,+T\left[\times\left(I_{j} \backslash\{0\}\right)\right.$, we have

$$
\int_{0}^{T} \int_{I_{j}}\left(\left|u_{j}-k\right| \frac{\partial \tilde{\varphi}}{\partial t}+\operatorname{sgn}\left(u_{j}-k\right)\left(f_{j}\left(u_{j}\right)-f_{j}(k)\right) \frac{\partial \tilde{\varphi}}{\partial x}\right) d x d t \geq 0 .
$$

(2) For every $j \in\{1, \ldots, n\}$ and for a.e. $t \in(0, T)$, the function $x \mapsto u_{j}(t, x)$ has a representation with bounded total variation.

(3) For a.e. $t>0$ we have

$$
\Psi\left(u_{1}(t, 0+), \ldots, u_{n}(t, 0+)\right)=0,
$$

where $u_{j}$ stands for the version with bounded total variation.

(4) For every $j \in\{1, \ldots, n\}, u_{j}(0, x)=u_{j, 0}$ for a.e. $x \in I_{j}$.

The construction of solutions will be based on the concept of Riemann solver at the node introduced in the following definition.

Definition 2.3. A Riemann solver $\mathcal{R} S$ is a function

$$
\mathcal{R} S:[0,1]^{n} \longrightarrow[0,1]^{n}, \quad\left(u_{1,0}, \ldots, u_{n, 0}\right) \longmapsto\left(\bar{u}_{1}, \ldots, \bar{u}_{n}\right),
$$

satisfying the following conditions:

(1) $\Psi\left(\bar{u}_{1}, \ldots, \bar{u}_{n}\right)=0$;

(2) for every $j \in\{1, \ldots, n\}$, the classical Riemann problem

$$
\left\{\begin{array}{l}
u_{t}+f_{j}(u)_{x}=0, \quad x \in \mathbb{R}, t>0, \\
u(0, x)= \begin{cases}\bar{u}_{j}, & \text { if } x<0, \\
u_{j, 0}, & \text { if } x>0,\end{cases}
\end{array}\right.
$$

is solved with waves with positive speed; 
(3) the consistency condition

$$
\mathcal{R} S\left(\mathcal{R} S\left(u_{1,0}, \ldots, u_{n, 0}\right)\right)=\mathcal{R} S\left(u_{1,0}, \ldots, u_{n, 0}\right)
$$

holds.

Remark 2.4. By Definition 2.3, a Riemann solver produces a solution to the Riemann problem (9), which respects the condition $\Psi\left(u_{1}(t, 0+), \ldots, u_{n}(t, 0+)\right)=0$. Moreover the image of a Riemann solver is the trace at $x=0$ of a solution to the Riemann problem; this fact is described by point 2 of Definition 2.3 , which prescribes that the generated waves have positive speed.

Now we state the three key properties of a Riemann solver, which will ensure the necessary bounds on approximate solutions (via wave-front tracking) and thus the existence of solutions to Cauchy problems. First we need some additional notation.

Definition 2.5. We say that $\left(u_{1,0}, \ldots, u_{n, 0}\right)$ is an equilibrium for the Riemann solver $\mathcal{R} S$ if

$$
\mathcal{R} S\left(u_{1,0}, \ldots, u_{n, 0}\right)=\left(u_{1,0}, \ldots, u_{n, 0}\right) .
$$

Definition 2.6. We say that a datum $u_{j} \in[0,1]$ is a good datum if either $u_{i} \in\left[0, \sigma_{i}\right]$ and $f_{i}$ is concave or $u_{i} \in\left[\sigma_{i}, 1\right]$ and $f_{i}$ is convex.

We say that a datum $u_{j} \in[0,1]$ is a bad datum if it not a good datum.

The first property requires that equilibria are determined only by bad data values, more precisely:

Definition 2.7. We say that a Riemann solver $\mathcal{R} S$ has the property (P1) if the following condition holds. Given $\left(u_{1,0}, \ldots, u_{n, 0}\right)$ and $\left(u_{1,0}^{\prime}, \ldots, u_{n, 0}^{\prime}\right)$ two initial data such that $u_{j, 0}=u_{j, 0}^{\prime}$ whenever either $u_{j, 0}$ or $u_{j, 0}^{\prime}$ is a bad datum, then

$$
\mathcal{R} S\left(u_{1,0}, \ldots, u_{n, 0}\right)=\mathcal{R} S\left(u_{1,0}^{\prime}, \ldots, u_{n, 0}^{\prime}\right) .
$$

The second property gives bounds on the increase of the flux variation for waves interacting with $J$. More precisely the latter should be bounded in terms of the strength of the interacting wave as well as the variation in the incoming fluxes.

Definition 2.8. We say that a Riemann solver $\mathcal{R} S$ has the property $(\mathrm{P} 2)$ if there exists a constant $C \geq 1$ such that the following condition holds. For every equilibrium $\left(u_{1,0}, \ldots, u_{n, 0}\right)$ of $\mathcal{R} S$ and for every wave $\left(u_{j, 0}, u_{j}\right)(j \in\{1, \ldots, n\})$ interacting with $J$ at time $\bar{t}>0$ and producing waves in the arcs according to $\mathcal{R} S$, we have

$$
\begin{aligned}
& \operatorname{TV}_{f}(\bar{t}+)-\operatorname{TV}_{f}(\bar{t}-) \\
& \leq C \min \left\{\left|f_{j}\left(u_{j, 0}\right)-f_{j}\left(u_{j}\right)\right|,\left|\sum_{f_{j}\left(\hat{u}_{j}\right)>0} f_{j}\left(\hat{u}_{j}\right)-\sum_{f_{j}\left(u_{j, 0}\right)>0} f_{j}\left(u_{j, 0}\right)\right|\right\},
\end{aligned}
$$

where we denoted by $\hat{u}_{j}$ the traces at $J$ of the waves produced by the Riemann solver $\mathcal{R}$. 
Remark 2.9. It is natural, for car traffic, to consider junctions composed by incoming and outgoing roads. Incoming roads can be described by the interval $(-\infty, 0)$, while the outgoing ones by the interval $(0,+\infty)$. In this situation the flux functions $f_{j}$ are all concave and positive. Therefore the terms $\sum_{f_{j}\left(u_{j, 0}\right)>0} f_{j}\left(u_{j, 0}\right)$ and $\sum_{f_{j}\left(\hat{u}_{j}\right)>0} f_{j}\left(\hat{u}_{j}\right)$, in vehicular traffic, correspond respectively to the sum of outgoing fluxes before and after the wave interaction. Consequently, in (10) of Definition 2.8, the term $\sum_{f_{j}\left(\hat{u}_{j}\right)>0} f_{j}\left(\hat{u}_{j}\right)-\sum_{f_{j}\left(u_{j, 0}\right)>0} f_{j}\left(u_{j, 0}\right)$ is the sum of the outgoing fluxes after the interaction minus the sum of outgoing fluxes before the interaction.

Now we state the property (P3): a wave interacting with $J$ and a flux decrease on a specific arc should also give rise to a decrease in the incoming fluxes.

Definition 2.10. We say that a Riemann solver $\mathcal{R} S$ has the property (P3) if, for every equilibrium $\left(u_{1,0}, \ldots, u_{n, 0}\right)$ of $\mathcal{R} S$ and for every wave $\left(u_{j, 0}, u_{j}\right)(j \in\{1, \ldots, n\})$ with $f_{j}\left(u_{j}\right)<f_{j}\left(u_{j, 0}\right)$ interacting with $J$ at time $\bar{t}>0$ and producing waves in the arcs according to $\mathcal{R} S$, we have

$$
\sum_{f_{j}\left(\hat{u}_{j}\right)>0} f_{j}\left(\hat{u}_{j}\right) \leq \sum_{f_{j}\left(u_{j, 0}\right)>0} f_{j}\left(u_{j, 0}\right),
$$

where we denoted by $\hat{u}_{j}$ the traces at $J$ of the waves produced by the Riemann solver $\mathcal{R S}$.

We also introduce the property (PS) of a Riemann solver, which guarantees that the solution of the Cauchy problem depends in a Lipschitz continuous way on the initial condition.

Definition 2.11. We say that a Riemann solver $\mathcal{R} S$ has the property (PS) if, for every equilibrium $\left(u_{1,0}, \ldots, u_{n, 0}\right)$ of $\mathcal{R} S$ and for every wave $\left(u_{j, 0}, u_{j}\right)(j \in\{1, \ldots, n\})$ interacting with $J$ at time $\bar{t}>0$ and producing waves in the arcs according to $\mathcal{R} S$, we have

$$
\operatorname{TV}_{f}(\bar{t}+) \leq \operatorname{TV}_{f}(\bar{t}-) .
$$

The main result is the following theorem.

Theorem 2.12. Consider the Cauchy problem (8) and a Riemann solver $\mathcal{R} S$ satisfying the properties (P1), (P2) and (P3). Assume that initial conditions $u_{j, 0}$ are bounded variation functions for every $j \in\{1, \ldots, n\}$.

Then there exists $\left(u_{1}(t, x), \ldots, u_{n}(t, x)\right)$, a weak solution at $J$, such that

(1) for every $j \in\{1, \ldots, n\}, u_{j}(0, x)=u_{j, 0}(x)$ for a.e. $x \in I_{j}$;

(2) for a.e.t $>0$,

$$
\mathcal{R} S\left(u_{1}(t, 0+), \ldots, u_{n}(t, 0+)\right)=\left(u_{1}(t, 0+), \ldots, u_{n}(t, 0+)\right) .
$$

If $\mathcal{R} S$ has the property (PS), then the solution $\left(u_{1}(t, x), \ldots, u_{n}(t, x)\right)$ depends in a Lipschitz continuous way on the initial condition (5). 
A proof of the previous result is in [72]. The existence of solutions part is based on the wave-front tracking technique (see [23], [71]); the key estimate consists in obtaining a uniform bound for the total variation of the flux of the approximate solutions. The Lipschitz continuity of the solution on the initial condition has been proved by using generalized differentials; see [24].

2.2. The system case. In this subsection, we deal with the case of systems. For simplicity, we consider only the case where $N_{j}=2$ for every $j \in\{1, \ldots, n\}$ and we assume that $\Omega_{j}$ is an open subset of $\mathbb{R}^{2}$ for every $j \in\{1, \ldots, n\}$.

We introduce classical assumptions on the flux functions.

(F) For every $j \in\{1, \ldots, n\}$, the flux $f_{j}$ is a smooth function such that:

(a) the Jacobian matrix $D f_{j}(u)$ is strictly hyperbolic for $u \in \Omega_{j}$, i.e. for every $u \in \Omega_{j}, D f_{j}(u)$ has two distinct real eigenvalues such that

$$
\lambda_{j, 1}(u)<\lambda_{j, 2}(u)
$$

with corresponding right eigenvectors $r_{j, 1}(u)$ and $r_{j, 2}(u)$;

(b) for every $i \in\{1,2\}$, the $i$-th characteristic field of $f_{j}$ is either genuinely nonlinear, i.e.

$$
\nabla \lambda_{j, i}(u) \cdot r_{j, i}(u) \neq 0 \quad \text { for all } u \in \Omega_{j},
$$

or linearly degenerate, i.e.

$$
\nabla \lambda_{j, i}(u) \cdot r_{j, i}(u)=0 \quad \text { for all } u \in \Omega_{j} .
$$

( $\Psi)$ We say that a function $\Psi \in \mathcal{C}^{1}\left(\prod_{j=1}^{n} \Omega_{j} ; \mathbb{R}^{n}\right)$ satisfies the assumption $(\Psi)$ at the point $\bar{u}=\left(\bar{u}_{1}, \ldots, \bar{u}_{n}\right) \in \prod_{j=1}^{n} \Omega_{j}$ if

$$
\operatorname{det}\left[D_{u_{1}} \Psi(\bar{u}) r_{2}(\bar{u}), \cdots D_{u_{1}} \Psi(\bar{u}) r_{2}(\bar{u})\right] \neq 0 \text {. }
$$

We have the following well-posedness result, whose proof is contained in [46].

Theorem 2.13. Let $n \in \mathbb{N}, n \geq 2$ and assume $(\mathrm{F})$. Fix an $n$-tuple of states $\bar{u}_{0} \in \Omega^{n}$ such that the Riemann problem (9) with initial datum $\bar{u}_{0}$ admits $u=\bar{u}_{0}$ as a solution to (9) in the sense of Definition 2.1. Let $\Psi$ satisfy assumption $(\Psi)$ at the point $\bar{u}_{0}$ and assume that $\lambda_{1}\left(\bar{u}_{1,0}\right)<0<\lambda_{2}\left(\bar{u}_{2,0}\right)$. Then there exist positive numbers $\delta, L$ and $a$ map $S:[0, \infty[\times \mathscr{D} \rightarrow \mathscr{D}$ such that

(1) $\mathscr{D} \supseteq\left\{u \in \bar{u}_{0}+L^{1}\left(\mathbb{R}^{+} ; \Omega^{n}\right): \operatorname{TV}(u) \leq \delta\right\}$;

(2) for $u \in \mathscr{D}, S_{0} u=u$ and for $s, t \geq 0, S_{s} S_{t} u=S_{s+t} u$;

(3) for $u, w \in \mathcal{D}$ and $s, t \geq 0,\left\|S_{t} u-S_{s} w\right\|_{L^{1}} \leq L\left(\|u-w\|_{L^{1}}+|t-s|\right)$;

(4) if $u \in \mathscr{D}$ is piecewise constant, then for $t>0$ sufficiently small, $S_{t} u$ coincides with the juxtaposition of the solutions to Riemann problems centered at the points of jumps or at the junction. 
Moreover, for every $u \in \mathcal{D}$, the map $t \mapsto S_{t} u$ is a solution to the Cauchy problem (7).

The proof is based on the wave-front tracking technique, which allows one the construction of approximate piecewise constant solutions. Bounds on the total variation allows one, through the Helly Compactness Theorem, to prove the existence of solutions. The use of Bressan-Liu-Yang functional [30] ensures that the approximate solutions and their limits are $L^{1}$ Lipschitz continuous with respect to the initial datum. Results of this type are local in the $u$-space, in the sense that the initial datum $u_{0}$ in (8) is required to satisfy

$$
u_{0} \in \bar{u}_{0}+L^{1} \quad \text { and } \quad \operatorname{TV}\left(u_{0}-\bar{u}_{0}\right)<\delta
$$

for a sufficiently small $\delta$. The requirement $(\Psi)$ at the node is a transversality condition between the gradient of $\Psi$ and the eigenvectors of $D f$. This condition, through an application of the Implicit Function Theorem, allows one to obtain suitable estimates on interactions at the nodal point. The case of systems with $N_{j}>2$ can be treated in a similar way. The fundamental assumptions are that the characteristic velocities at $\bar{u}_{0}$ are all different from 0 and that the function $\Psi$ satisfies a suitable transversality condition at the point $\bar{u}_{0}$.

Remark that the introduction of a source term typically makes it impossible to obtain global in time existence results. In general, without any invariance condition, the source term may cause a drift of the solution in the $u$-space, exiting the neighborhood of $u^{*}$ where the Implicit Function Theorem can be applied and hence Riemann problems can be solved.

\section{Models}

Several "real situations" are described by nonlinear hyperbolic systems of balance laws on networks. Among them we recall: traffic flow, gas pipelines, supply chain models, telecommunication networks, systems of open canals and blood flow in arteries.

For all the previous situations, we present a rigorous mathematical description, including both the system considered in each arc of the network and the coupling conditions at junctions. Clearly both the systems and the coupling conditions depend on the applications and they have been derived according to physical and modeling considerations. Since the section is devoted to models, no theoretical result is presented here.

3.1. Traffic flow on road networks. The use of conservation laws in the modeling of traffic dynamics goes back to the pioneering works of Lighthill-Whitham [109] 
and Richards [117], introducing the (LWR) model

$$
\partial_{t} \rho(t, x)+\partial_{x} f(\rho(t, x))=0
$$

where $\rho \in\left[0, \rho_{\max }\right]$ is the density of cars at time $t$ and at position $x, f(\rho)=\rho v(\rho)$ is the flux and $v$ is a given function depending only on the density. Typically, the flux $f$ is a $\ell^{2}$ concave function such that $f(0)=f\left(\rho_{\max }\right)=0$. Without loss of generality, we assume that $\rho_{\max }=1$. The case of car traffic on network has been considered in various papers; see for instance [41], [95], [100], [71].

In traffic, it is customary to define a junction by a finite number of incoming and outgoing roads. Incoming roads are described by the half line $(-\infty, 0)$, while outgoing roads by the interval $(0,+\infty)$. Clearly with the position $x \mapsto-x$ the incoming roads can be represented by the interval $(0,+\infty)$, fitting the notations of this paper. For a junction with $m_{1}$ incoming roads and $m_{2}$ outgoing ones, one is thus lead to consider the system

$$
\left\{\begin{array}{l}
\partial_{t} \rho_{j}+\partial_{x} f\left(\rho_{j}\right)=0, \quad x \in \mathbb{R}^{-}, j \in\left\{1, \ldots, m_{1}\right\} \\
\partial_{t} \rho_{j}+\partial_{x} f\left(\rho_{j}\right)=0, \quad x \in \mathbb{R}^{+}, j \in\left\{m_{1}+1, \ldots, m_{1}+m_{2}\right\}
\end{array}\right.
$$

At the nodal point $x=0$, one prescribes the conservation of cars, which reads

$$
\sum_{j=1}^{m_{1}} f\left(\rho_{j}(t, 0)\right)=\sum_{j=m_{1}+1}^{m_{1}+m_{2}} f\left(\rho_{j}(t, 0)\right) .
$$

This is not sufficient to isolate a unique solution to the Riemann problem at the node; hence one should consider other additional rules. One possibility is to also consider first the preference of drivers and then the maximization of the flux through the junction. The preference of drivers can be described by introducing a Markov matrix

$$
A=\left(\begin{array}{ccc}
\alpha_{m_{1}+1,1} & \cdots & \alpha_{m_{1}+m_{2}, 1} \\
\vdots & \ddots & \vdots \\
\alpha_{m_{1}+1, m_{1}} & \cdots & \alpha_{m_{1}+m_{2}, m_{1}}
\end{array}\right)
$$

whose entries $\alpha_{j, i} \in[0,1]$ are the percentage of traffic coming from the $i$-th incoming road and going to the $j$-th outgoing road. Therefore, if we denote by $\gamma_{j}$ the flux exiting the $j$-th incoming road or the flux entering the $j$-th outgoing road, then we have

$$
\left(\gamma_{m_{1}+1}, \ldots, \gamma_{m_{1}+m_{2}}\right)^{\top}=A \cdot\left(\gamma_{1}, \ldots, \gamma_{m_{1}}\right)^{\top}
$$

Imposing also the maximization of the flux through the junction, i.e.

$$
\max \left(\gamma_{1}+\cdots+\gamma_{m_{1}}\right)
$$


the Riemann problem at the node admits a unique solution; see [41]. In [41], it is also proved that the Cauchy problem for (12) with nodal conditions (13), (14) and (15) admits an entropy admissible solution, defined for every time interval $[0, T]$. In general the solution does not depend in a Lipschitz continuous way on the initial condition. A counterexample is given in [41].

It is interesting to note that the existence and well-posedness properties for the Cauchy problem associated to (12) depend on the choice of the nodal conditions; see [72].

A different approach, based on a vanishing viscosity approximation, to the Cauchy problem for (12) was developed in [40].

In case of high traffic, the LWR model is not so satisfactory; hence one can consider a model of the second order on the network, for example the Aw-Rascle model or a phase-transition one. The Aw-Rascle model [8] is defined by

$$
\left\{\begin{array}{l}
\partial_{t} \rho+\partial_{x}\left(y-\rho^{\gamma+1}\right)=0 \\
\partial_{t} y+\partial_{x}\left(\frac{y^{2}}{\rho}-y \rho^{\gamma}\right)=0 .
\end{array}\right.
$$

In (16), $\rho$ is the density of the cars, $y=\rho v+\rho^{\gamma+1}$ is a generalized momentum, $v$ is the velocity of the cars and $\gamma>0$ is a constant. Therefore the complete system reads

$$
\left\{\begin{array}{l}
\left\{\begin{array}{l}
\partial_{t} \rho_{j}+\partial_{x}\left(y_{j}-\rho_{j}^{\gamma+1}\right)=0, \\
\partial_{t} y_{j}+\partial_{x}\left(\frac{y_{j}^{2}}{\rho_{j}}-y_{j} \rho_{j}^{\gamma}\right)=0,
\end{array} \quad x<0, j \in\left\{1, \ldots, m_{1}\right\},\right. \\
\left\{\begin{array}{l}
\partial_{t} \rho_{j}+\partial_{x}\left(y_{j}-\rho_{j}^{\gamma+1}\right)=0, \\
\partial_{t} y_{j}+\partial_{x}\left(\frac{y_{j}^{2}}{\rho_{j}}-y_{j} \rho_{j}^{\gamma}\right)=0,
\end{array} \quad x>0, j \in\left\{m_{1}+1, \ldots, m_{1}+m_{2}\right\} .\right.
\end{array}\right.
$$

Similar to the LWR case, it is possible to impose nodal conditions which prescribe the conservation of the number of cars, the preference of drivers and the maximization of the flux passing through the junction. In [70], it is shown that the previous conditions are not sufficient to isolate a unique solution on the outgoing roads. Hence three different additional rules have been considered in [70]: The minimization of the density, the maximization of the speed of cars and the minimization of the total variation of the solution. Each of the previous rules permits to define a well-defined Riemann solver and the corresponding Cauchy problem admits a solution which is defined globally in time, but locally in space.

Different coupling conditions have been presented in [88] using homogenization methods on the outgoing arcs. This approach is specially designed for the AwRascle-Zhang model and uses the fact that the second conservation law for $y$ may be reformulated as

$$
y_{t}+v y_{x}=0 .
$$


The phase transition model, defined independently in [19], [48], has the form

$$
\begin{cases}\partial_{t} \rho+\partial_{x}(\rho V)=0 & \text { if }(\rho, q) \in \Omega_{f}, \\
\left\{\begin{array}{l}
\partial_{t} \rho+\partial_{x}\left(\rho v_{c}(\rho, q)\right)=0, \\
\partial_{t} q+\partial_{x}\left(q v_{c}(\rho, q)\right)=0,
\end{array}\right. & \text { if }(\rho, q) \in \Omega_{c},\end{cases}
$$

where $\Omega_{f}$ and $\Omega_{c}$ denote respectively the free phase and the congested one, $\rho$ is the density of cars, $V$ is the maximal velocity, $v_{c}$ is the speed of cars in $\Omega_{c}$ and $q$ is a "generalized" momentum. With the same technique as in [43], it is possible to consider the Cauchy problem for the phase-transition model on a junction and to find a solution to that Cauchy problem.

3.2. Gas dynamics in pipelines. Historically, Euler equations of gas dynamics have been the paradigm according to which the theory of conservation (or balance) laws has been developed. Also in the case of nodal points, (high-pressure) gas pipelines provide stimulating real problems, see for instance [11], [10], [42], [45], [46], [49], [81], [96].

In a typical real pipe the ratio between pipe length and diameter is large enough to justify the use of Euler equations in one space dimension, or approximations thereof. Therefore, the natural setting for gas pipeline models consists of $n$ pipes connected at a fixed point, say $x_{*}=0$, and the gas is described through the isentropic Euler equations so that (1) consists of $n$ copies of the $p$-system, i.e., in (6) we set

$$
\begin{gathered}
u_{j}=\left[\begin{array}{l}
\rho_{j} \\
q_{j}
\end{array}\right], \quad f_{j}(u)=\left[\begin{array}{c}
q_{j} \\
\frac{q_{j}^{2}}{\rho_{j}}+p\left(\rho_{j}\right)
\end{array}\right], \\
g_{j}(t, x, u)=\left[\begin{array}{c}
0 \\
-v \frac{q_{j}\left|q_{j}\right|}{\rho_{j}}-\rho_{j} \bar{g} \sin \alpha_{j}(x)
\end{array}\right],
\end{gathered}
$$

where the pressure law $p$ can be, for instance, the usual $\gamma$-law $p(\rho)=\kappa \rho^{\gamma}$. Above, $g_{j}$ is a typical source term with a zero component in the mass equation, while the second component describes the effect $\bar{g} \sin \alpha_{j}(x)$ of gravity $\left(\alpha_{j}(x)\right.$ being the slope of the $j$-th pipe at $x$ ) and the effect $-v q|q| / \rho$ of friction ( $v$ being a constant parameter) on the balance of momentum. Remark that in the case $n=2$ the present setting may also describe the dynamics of gas flowing in a pipe with a kink [96].

The 1D framework allows a rather simple modeling structure and, mostly, very fast numerical integration. A model in one space dimension well describes the dynamics within a pipe, but hardly covers geometry effects at a junction, which is clearly a familiar 3D phenomenon. As a consequence, the literature offers several different choices for the nodal condition (2), depending on the specific needs of each particular situation. In the engineering literature, the nodal conditions are typically supplied 
with parameters whose values are empirically justified. A numerical study of oneand two-dimensional situations can be found in [84], [91].

In the subsonic setting, condition (2) has to provide $n$ conditions to single out a unique solution to the Riemann problem (7). One component of $\Psi$, say the first,

$$
\Psi_{1}(u)=\sum_{j=1}^{n} a_{j} q_{j}
$$

ensures the conservation of mass. Here, $a_{j}$ is the surface section of the $j$-th pipe.

The other components of $\Psi$ may impose, for instance, equal pressure at the node,

$$
\Psi_{j}(u)=p\left(\rho_{j}\right)-p\left(\rho_{1}\right), \quad j=2, \ldots, n,
$$

or the continuity of the dynamic pressure at the node,

$$
\Psi_{j}(u)=a_{j}\left(\frac{q_{j}^{2}}{\rho_{j}}+p\left(\rho_{j}\right)\right)-a_{1}\left(\frac{q_{1}^{2}}{\rho_{1}}+p\left(\rho_{1}\right)\right), \quad j=2, \ldots, n .
$$

We refer to [11], [10], [42], [46] and to the references therein for more details on the related modeling and computational results. On the other hand, the nodal point may well be a model for a compressor station between $n=2$ pipes having the same section $a_{1}=a_{2}$. In this case, the nodal condition consists of (17), while (18) is replaced, for instance, by

$$
\Psi_{2}(t, u)=q_{2}\left(\left(\frac{p\left(\rho_{2}\right)}{p\left(\rho_{1}\right)}-1\right)^{(\gamma-1) / \gamma}\right)-\Pi(t)
$$

see Section 3.1 in [45], Formula (4.9) in Section 4.4 of [110] or [119]. Here, $\Pi$ is proportional to the power exerted by the compressor. This framework naturally leads to various control problems, where the open-loop control $\Pi=\Pi(t)$ has to be chosen to satisfy suitable optimality criteria, see [15], [79], [45], [63], [81]. Closed-loop control problems, where $\Pi$ depends on the traces $u_{1}\left(t, x_{*}+\right)$ and $u_{2}\left(t, x_{*}+\right)$ of the state of the fluid, were studied in the case of smooth solutions in [62], [81].

For completeness, we recall that the above has been partly extended to the case of the full $3 \times 3$ system of Euler equations in [47], [49].

A drift-flux model for a two-phase gas was considered in [13] in the isothermal case under a no-slip assumption. Here, (1) and (2) can be used, with (6) and

$$
u_{j}=\left[\begin{array}{c}
\rho_{j}^{1} \\
\rho_{j}^{2} \\
\left(\rho_{j}^{1}+\rho_{j}^{2}\right) v_{j}
\end{array}\right], \quad f_{j}\left(u_{j}\right)=\left[\begin{array}{c}
\rho_{j}^{1} v \\
\rho_{j}^{2} v \\
\left(\rho_{j}^{1}+\rho_{j}^{2}\right)\left(\left(v_{j}\right)^{2}+\frac{a^{2}}{2}\right)
\end{array}\right], \quad g(t, x, u)=0 .
$$

Here, $\rho_{j}^{i}$ is the density of the $i$-th component in the $j$-th pipe and $v_{j}$ is the fluid speed, common to both phases in the $j$-th pipe. The sound speed $a$ is assumed to be the 
same for all phases, see [12] for a more general setting. At the junction, the nodal condition (2) imposes the conservation of the total mass of each phase, similarly to (17). Besides, it is usually required that all velocity flows at the junction are equal so that

$$
\Psi_{j}(u)=\frac{1}{2}\left(v_{j}^{2}-v_{1}^{2}\right)+\frac{a^{2}}{2} \log \frac{\rho_{j}^{1}+\rho_{j}^{2}}{\rho_{1}^{1}+\rho_{1}^{2}}, \quad j=2, \ldots, n .
$$

The existence of weak solutions to (1), (2), (17) and (20) for constant initial data with separated wave speeds was obtained in Proposition 3.1 of [13].

In order to capture effects of the 3D situation, numerical integration [84], [91] of multi-dimensional formulation of gas dynamics close to a nodal point have been performed. A local zooming has been introduced and a 2D domain $\mathscr{D}$ models the area at the nodal point. Then, for $(x, y) \in \mathscr{D}$ and the usual $\gamma$-law for $p$ the isentropic Euler equations

$$
\partial_{t}\left(\begin{array}{c}
\rho \\
\rho u \\
\rho v
\end{array}\right)+\partial_{x}\left(\begin{array}{c}
\rho u \\
\rho u^{2}+p(\rho) \\
\rho u v
\end{array}\right)+\partial_{y}\left(\begin{array}{c}
\rho v \\
\rho u v \\
\rho v^{2}+p(\rho)
\end{array}\right)=0
$$

are solved numerically. For piecewise constant initial data comparisons of flow and pressure computations by nodal conditions (17)-(18) and (17)-(19) with numerical averaging of solutions $(\rho, \rho u, \rho v)$ of (21) have been studied [91]. For initial data and small velocities $v_{j, 0}$ the results should have qualitatively a similar behavior. Further studies exist for the Euler equations [84].

3.3. Product flow in supply chains. A continuum description of the product density in high-volume production lines has been derived and analyzed [6]. The governing equations are of the type (1), where

$$
u=\left[\begin{array}{c}
u_{1} \\
\vdots \\
u_{n}
\end{array}\right], \quad f(u)=\left[\begin{array}{c}
f_{1}\left(u_{1}\right) \\
\vdots \\
f_{n}\left(u_{n}\right)
\end{array}\right] \text { and } f_{j}\left(u_{j}\right)=\min \left\{v_{j} u_{j}, \mu_{j}\right\}
$$

Here, the index $j$ refers to the supplier, $u_{j}$ is the product density; $v_{j}$, respectively $\mu_{j}$, is the non-negative constant production velocity, respectively capacity, see [7], [76], [77]. Similarly to traffic flow networks, we distinguish between the $l$ suppliers delivering to the nodal point at $x_{*}=0$ and the $n-l$ suppliers receiving goods from $x_{*}$. But differently from what happens in traffic modeling, due to possible differences in the total capacities of the different suppliers, the total mass is typically not conserved at the node. Therefore, the dynamics of the suppliers $1, \ldots, l$ is coupled through a priori unknown new functions $q_{1}(t), \ldots, q_{l}(t)$ describing the amount of goods waiting in suitable buffers when the lines they have to enter are congested. This leads 
to a time-dependent coupling condition of the type (2), namely

$$
\Psi(t, u)=\int_{t_{0}}^{t}\left(\sum_{j=1}^{l} f_{j}\left(u_{j}(s, 0-)\right)-\sum_{j=l+1}^{n} f_{j}\left(u_{j}(s, 0+)\right)\right) d s-q_{j}(t)+q_{j}\left(t_{0}\right) .
$$

Clearly, (23) is not sufficient to obtain a well-posed Riemann problem at the node and therefore is complemented by the $j=l+1, \ldots, n$ coupling conditions

$$
\Psi_{j}(t, u)=q_{j}(t)-q_{j}\left(t_{0}\right)+\int_{t_{0}}^{t}\left(f_{j}\left(u_{j}(s, 0+)\right)-\sum_{k=1}^{l} \alpha_{j k}(s) f_{k}\left(u_{k}(s, 0-)\right)\right) d s
$$

that prescribe how the goods are distributed among the outgoing lines. Indeed, the known parameter $\alpha_{j k}(t) \in[0,1]$ is the portion of good flowing from the $k$-th line that have to enter the $j$-th one at time $t$, for $k=1, \ldots, l$ and $j=l+1, \ldots, n$.

The well-posedness of the Riemann problem (7), (22)-(24), as well as that of the corresponding Cauchy problem, is proved in [56], [87]. An extension of the model (22) has been proposed in [57], [60] to treat the case of supply chains with spatially and temporally depending capacities. Therein, $\mu_{j}=\mu_{j}(t, x)$ and equation (22) is replaced by $u_{j}=\left(\rho_{j}, \mu_{j}\right)$ and $f_{j}(u)=((22),-\mu)$, respectively. Coupling conditions of the form (2) are proposed conserving total mass and the value of $\mu$. Existence of solutions to the Cauchy problem for initial data with zero total variation in $\mu$ has been established in Theorem 3 of [57].

3.4. Data flow on telecommunication networks. The transport of data packages on internet is modeled by using conservation laws on networks [58], [59]. Each connection between two servers corresponds to an edge connecting two nodal points. In each line, the transmission of packets can be described by the scalar conservation law

$$
\partial_{t} \rho+\partial_{x} f(\rho)=0,
$$

where $\rho=\rho(t, x)$ is the density of packets and

$$
f(\rho)= \begin{cases}\bar{v} \rho, & 0 \leq \rho \leq \sigma, \\ \bar{v} \sigma \frac{\rho_{\max }-\rho}{\rho_{\max }-\sigma}, & \sigma \leq \rho \leq \rho_{\max }\end{cases}
$$

Here $\bar{v}$ is the constant velocity of the packets, $\rho_{\max }$ is the maximum density and $\sigma \in\left(0, \rho_{\max }\right)$ is a parameter, which is related to the probability of packets loss. The model (25) on a junction composed by $m_{1}$ incoming transmission line and $m_{2}$ outgoing ones is considered in [59].

At the nodal point the Riemann solver is defined according to the following rules:

(1) the flux passing through the junction is the maximal possible; 
(2) once the flux through the junction is determined, then priority coefficients distribute the flux among the various transmission lines.

A proof for existence of the corresponding Cauchy problem is given in [59].

3.5. Networks of open canals. Water flow in open canals [16], [79], [102] can be described by the Saint-Venant equations in one space dimension, which read as

$$
\left\{\begin{array}{l}
\partial_{t} a+\partial_{x}(v a)=0 \\
\partial_{t} v+\partial_{x}\left(\frac{v^{2}}{2}+g H(a)\right)=-S(a, v) .
\end{array}\right.
$$

Here, $a$ is the wet cross section, $v$ is the velocity, $g$ is the gravity constant, $H=H(a)$ is the water height corresponding to $a$ and $S=S(a, v)$ is a source term, which takes care of the slope of the channel and of the friction due to the slope. At a junction between $n$ canals, we are lead to consider $n$ copies of (26), with unknowns $a_{j}$ and $v_{j}$, coupled with the nodal condition

$$
\left\{\begin{array}{l}
\sum_{j=1}^{n} a_{j} v_{j}=0, \\
\frac{1}{2} v_{j}^{2}+\bar{g} h\left(a_{j}\right)=\frac{1}{2} v_{1}^{2}-\bar{g} h\left(a_{1}\right), \quad j=2, \ldots, n,
\end{array}\right.
$$

see [46], [102], ensuring the conservation of water and the Bernoulli law to be satisfied at the junction.

3.6. The arterial network. Blood flow through the arterial network can be modeled by the following reduced, 1D hyperbolic system [34]:

$$
\left\{\begin{array}{l}
\partial_{t} a+\partial_{x}(a v)=0 \\
\partial_{t}(a v)+\partial_{x}\left(\alpha a v^{2}\right)+\frac{a}{\rho} \partial_{x} p(a)=-2 \frac{\alpha}{\alpha-1} \nu v
\end{array}\right.
$$

Here, $x$ denotes the distance along the center line of the artery (see Figure 2), $a=a(x, t)$ is the (scaled) cross-sectional vessel area at position $x$ and time $t$, $v=v(x, t)$ is the cross-sectional average fluid velocity, $v$ is the kinematic viscosity coefficient ( $v \approx 3.2 \times 10^{-6} \mathrm{~m}^{2} / \mathrm{s}$ for blood), and $\alpha$ is the so called "Coriolis coefficient" [65], or the correction factor that takes into account the fact that the resulting equation models the conservation of average momentum, and not the actual momentum. It is associated with the closure problem, typical for dimension reduction in nonlinear problems [34]. The value of $\alpha$ is associated with the assumption on the horizontal velocity profile for this model. If one assumes the Poiseuille flow (quadratic velocity profile associated with Newtonian fluids), we obtain $\alpha=4 / 3$. For the plug velocity profile (associated with the non-Newtonian nature of blood flow), $\alpha=11 / 10$.

In this model $p=p(a)$ denotes the fluid pressure, which is a function of the cross-sectional area $a$. The relationship between $p$ and $a$ specifies the mechanical 


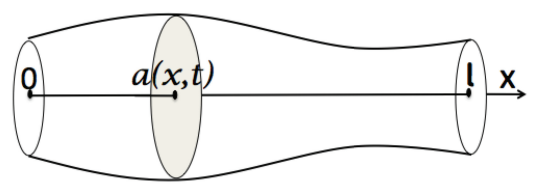

Figure 2. A sketch of an artery and cross-sectional area in equations (27).

properties of arterial walls (constitutive relation). Typically, the linear elastic membrane equation, also known as the Law of Laplace, is assumed:

$$
p(a)=p_{\text {ref }}+\frac{h E}{\left(1-\sigma^{2}\right) R_{0}}\left(\sqrt{\frac{a}{a_{0}}}-1\right)=p_{\text {ref }}+\beta\left(\sqrt{\frac{a}{a_{0}}}-1\right),
$$

where $R_{0}$ is the radius of the reference cylinder, $a_{0}=R_{0}^{2}, h$ is the thickness of the arterial wall (membrane thickness), $E$ the Young's modulus of elasticity, and $\sigma$ is the Poisson ratio. We denoted by $p_{\text {ref }}$ the reference (external) pressure, namely, the pressure at which the displacement of the arterial wall is zero, i.e., the cross-sectional area $a=a_{0}$. For the corresponding nonlinear membrane model see [37].

Equations (27) can be derived from the full set of Navier-Stokes equations for an incompressible, viscous fluid, by assuming axial symmetry of the domain and of the flow. They are obtained after averaging the full 3D axially symmetric Navier-Stokes equations, and assuming an ad hoc closure for the form of the axial velocity profile. The resulting equations (27) are not in conservation form.

By assuming smooth solutions, system (27) is equivalent to the following system written in conservation form:

$$
\left\{\begin{array}{l}
\partial_{t} a+\partial_{x} q=0, \\
\partial_{t} q+\partial_{x}\left(\alpha \frac{q^{2}}{a}+\frac{1}{\rho} \pi(a)\right)=-2 \frac{\alpha}{\alpha-1} \nu \frac{q}{a},
\end{array}\right.
$$

where $q=a v$ is the fluid momentum of the averaged problem, and

$$
\pi(a)=\int_{a_{0}}^{a} \tilde{a} p^{\prime}(\tilde{a}) d \tilde{a} .
$$

The conservation of mass principle for the full 3D problem translates into the conservation of "volume" (quantity $a$ ), described by the first equation in (29), while the averaged fluid momentum $q$ satisfies a balance law (the second Newton law of motion) for the average problem, described by the second equation in (29). We emphasize again that for solutions involving jump discontinuities, systems (27) and (29) are not equivalent. 
A further simplification, assuming smooth solutions, leads to the system

$$
\left\{\begin{array}{l}
\partial_{t} a+\partial_{x} a v=0 \\
\partial_{t} v+\partial_{x}\left(\alpha \frac{v^{2}}{2}+\frac{1}{\rho} \pi(a)\right)=-2 \frac{\alpha}{\alpha-1} v v
\end{array}\right.
$$

which is the Saint-Venant system (26). For $\alpha=1$ the quantity $\frac{v^{2}}{2}+\frac{1}{\rho} \pi(a)$ under the spatial derivative in the second equation corresponds to the scaled total (or dynamic) pressure by the constant density $\rho$.

Each of the systems (27), (29) or (30), describes the flow of blood in a single vessel. For a network problem, system (27), (29) or (30) holds for each artery meeting at a given node. Suppose for a moment, that system (27) is used to describe blood flow for each artery meeting at a node. To study the interaction of waves described by equations (27) at a node, the equations holding along each branch are coupled at the node by imposing some physically reasonable coupling conditions. The typical ones are:

- conservation of mass at the node, and

- continuity of pressure (or even total pressure) at the node.

Suppose that there are $n$ branches meeting at a node. The continuity of mass flux at a node is then given by

$$
\sum_{j=1}^{n}( \pm 1) a_{j} v_{j}=0
$$

where the plus or minus sign in (31) depends on the parametrization of the incoming/outgoing branches. The parametrization typically reflects the direction of flow.

Regarding the continuity of pressure (or total pressure) at the node, two different coupling conditions related to the behavior of the pressure/total pressure at the branching point have been used in literature. One describes the continuity of pressure $p$ appearing in equation (27) [114]:

$$
p_{1}=p_{j}, \quad j=2, \ldots, n,
$$

while the other, used in e.g., [66], requires continuity of the total flux in the second equation of (30), (or, equivalently, the flux in (29)), which describes the continuity of total pressure at the node:

$$
\alpha \rho \frac{v_{1}^{2}}{2}+\pi\left(a_{1}\right)=\alpha \rho \frac{v_{j}^{2}}{2}+\pi\left(a_{j}\right), \quad j=2, \ldots, n .
$$

This condition corresponds to the second coupling condition in the Saint-Venant model (26). Rewritten in terms of the state variables $(a, q)$ appearing in problem (29) 
this condition reads

$$
\alpha \rho \frac{q_{1}^{2}}{2 a_{1}}+\pi\left(a_{1}\right)=\alpha \rho \frac{q_{j}^{2}}{2 a_{j}}+\pi\left(a_{j}\right), \quad j=2, \ldots, n .
$$

Thus, the following two sets of coupling conditions have been used in hemodynamics literature:

$$
\left\{\begin{array}{l}
\sum_{j=1}^{n} \pm a_{j} v_{j}=0, \\
p_{1}=p_{j}
\end{array}\right\}, \quad\left\{\begin{array}{l}
\sum_{j=1}^{n} \pm a_{j} v_{j}=0 \\
\alpha \rho \frac{v_{1}^{2}}{2}+\pi\left(a_{1}\right)=\alpha \rho \frac{v_{j}^{2}}{2}+\pi\left(a_{j}\right)
\end{array}\right\} .
$$

\section{Model fusion}

In recent years a significant effort was devoted by applied mathematicians to the modeling of "real systems" by partial differential equations, thus dealing with the macroscopic scale of the problem. Such effort enriched the already wide spectrum of available mathematical models, many of which were focused on the microscopic scale. Therefore researchers and practitioners face now an unprecedented opportunity in model choice. In turn this poses the problem of identifying the "best" model. It is common opinion that different mathematical frameworks present different characteristics, thus the best modeling approach is obtained by combining more than one model.

A typical situation is that of a large complex road network, with a small portion of it requiring a fine modeling and the remaining part for which a coarse model is sufficient. This leads to the necessity of combining different models using coupling conditions at interfaces.

In this section we present two different situations. The first one consists in a mixed system, composed by ODEs and PDEs with a moving boundary; the second one deals with the coupling, at a fixed boundary, of two different macroscopic traffic models. In both cases, we present both the mathematical description and a theoretical result about existence of solutions for the Cauchy problem.

4.1. Mixed ODEs-PDEs Systems. When nodal conditions are coupled with ordinary differential equations, the conditions allowing the well-posedness are more intricate. Moreover, key questions about the existence of solutions globally in time remain mostly unanswered. 
Let us consider the following mixed systems, composed by hyperbolic balance laws and ordinary differential equations:

$$
\left\{\begin{array}{l}
\partial_{t} u+\partial_{x} f(u)=g(u), \quad x>\gamma(t), \\
b(u(t, \gamma(t)+))=B(t, w(t)), \\
\dot{w}=F(t, u(t, \gamma(t)+), w(t)) \\
\dot{\gamma}(t)=\Pi(w(t))
\end{array}\right.
$$

Here the unknowns are $u=u(t, x), w=w(t)$ and $\gamma=\gamma(t) . u$ is defined for $t \geq 0$ and $x \geq \gamma(t), w$ for $t \geq 0$ and $\gamma$, the boundary for the partial differential equation, for $t \geq 0$. Let $\Omega \subseteq \mathbb{R}^{n}$ be an open set and fix the reference states $\hat{u} \in \Omega, \hat{w} \in \mathbb{R}^{m}$ and $\hat{x} \in \mathbb{R}$. For $\delta>0$, define the sets

$$
\begin{aligned}
\mathcal{U} & =\left\{u \in \hat{u}+\left(\mathrm{BV} \cap L^{1}\right)\left(\mathbb{R} ; \mathbb{R}^{n}\right): u(\mathbb{R}) \subset \Omega\right\}, \\
\mathcal{U}_{\delta} & =\{u \in \mathcal{U}: \operatorname{TV}(u) \leq \delta\} .
\end{aligned}
$$

In order to present the well-posedness for (32), we introduce the following conditions.

(f) $f \in \mathcal{C}^{4}\left(\Omega ; \mathbb{R}^{n}\right)$ is smooth and such that, for all $u \in \Omega, D f(u)$ is strictly hyperbolic and each characteristic field is either genuinely nonlinear or linearly degenerate.

For $u \in \Omega$ and $i=1, \ldots, n$, call $\lambda_{i}(u)$ the $i$-th eigenvalue of $D f(u)$ and $r_{i}(u)$ the corresponding right eigenvector. By (f), we may assume that $\lambda_{i-1}(u)<\lambda_{i}(u)$ for all $u \in \Omega$ and $i=2, \ldots, n$.

(g) For $\delta>0, g: U_{\delta} \rightarrow L^{1}\left(\mathbb{R} ; \mathbb{R}^{n}\right)$ is such that for suitable $L_{1}, L_{2}>0$,

$$
\left\|g(u)-g\left(u^{\prime}\right)\right\|_{L^{1}} \leq L_{1}\left\|u-u^{\prime}\right\|_{L^{1}} \quad \text { and } \operatorname{TV}(g(u)) \leq L_{2}
$$

for all $u, u^{\prime} \in U_{\delta}$.

(I) $\Pi \in e^{0,1}\left(\mathbb{R}^{m} ; \mathbb{R}\right)$.

(NC) There exist $c>0$ and $\ell \in\{1,2, \ldots, n-1\}$ such that $\lambda_{\ell}(\hat{u})<\Pi(\hat{w})-c$ and $\lambda_{\ell+1}(\hat{u})>\Pi(\hat{w})+c$.

The above non-characteristic (NC) condition on $f$ is coordinated with the following assumption on $b$, which describes how the boundary data are assigned.

(b) $b \in \mathcal{C}^{1}\left(\Omega ; \mathbb{R}^{n-\ell}\right)$ is such that

$$
\operatorname{det}\left(D_{u} b(\hat{u})\left[r_{\ell+1}(\hat{u}) \quad r_{\ell+2}(\hat{u}) \quad \cdots \quad r_{n}(\hat{u})\right]\right) \neq 0 .
$$

Condition (b) is the assumption on the assignment of boundary data in a noncharacteristic problem for a conservation law, see for instance [2], [3], [44], [118]. Besides, it imposes $b$ to be not invertible. The case of an invertible $b$ would formally correspond to $\ell=0$ in (b) and would allow the decoupling of system (32) in a PDE and a separate ODE. 
(F) The map $F: \mathbb{R}^{+} \times \Omega \times \mathbb{R}^{m} \longrightarrow \mathbb{R}^{m}$ is subject to the following conditions:

(F.1) For all $u \in \Omega$ and $w \in \mathbb{R}^{m}$, the function $t \mapsto F(t, u, w)$ is Lebesgue measurable.

(F.2) For every compact subset $K$ of $\Omega \times \mathbb{R}^{m}$, there exists $C_{K}>1$ such that for all $t \in \mathbb{R}^{+}$and $\left(u_{1}, w_{1}\right),\left(u_{2}, w_{2}\right) \in K$

$$
\left\|F\left(t, u_{1}, w_{1}\right)-F\left(t, u_{2}, w_{2}\right)\right\|_{\mathbb{R}^{m}} \leq C_{K}\left(\left\|u_{1}-u_{2}\right\|_{\mathbb{R}^{n}}+\left\|w_{1}-w_{2}\right\|_{\mathbb{R}^{m}}\right) .
$$

(F.3) There exists a function $C \in L_{\text {loc }}^{1}\left(\mathbb{R}^{+} ; \mathbb{R}^{+}\right)$such that for all $t>0, u \in \Omega$ and $w \in \mathbb{R}^{m}$

$$
\|F(t, u, w)\|_{\mathbb{R}^{m}} \leq C(t)\left(1+\|w\|_{\mathbb{R}^{m}}\right) .
$$

(B) $B \in \mathcal{C}^{1}\left(\mathbb{R}^{+} \times \mathbb{R}^{m} ; \mathbb{R}^{n-\ell}\right)$ is locally Lipschitz, i.e., for every compact subset $K$ of $\mathbb{R}^{m}$ there exists a constant $\widetilde{C}_{K}>0$ such that, for every $t>0$ and $w \in K$,

$$
\left\|\frac{\partial}{\partial t} B(t, w)\right\|_{\mathbb{R}^{n-\ell}}+\left\|\frac{\partial}{\partial w} B(t, w)\right\|_{\mathbb{R}^{n-\ell}} \leq \widetilde{C}_{K} .
$$

Now we pass to the problem (32), providing a rigorous definition of its solution.

Definition 4.1. Let $T>0$. A triple $(u, w, \gamma)$ with

$$
u \in \mathcal{C}^{0}([0, T] ; U), \quad w \in W^{1,1}\left([0, T] ; \mathbb{R}^{m}\right), \quad \gamma \in W^{1, \infty}\left([0, T] ; \mathbb{R}^{m}\right)
$$

is a solution to (32) on $[0, T]$ with initial datum $\left(u_{0}, w_{0}, x_{0}\right)$ such that $u_{0} \in \mathcal{U}$ with $u_{0}(x)=\hat{u}$ for $x<x_{0}, w_{0} \in \mathbb{R}^{m}$ and $x_{0} \in \mathbb{R}$, if

(1) $u$ is an entropy admissible solution to

$$
\left\{\begin{array}{l}
\partial_{t} u+\partial_{x} f(u)=g(u), \quad x>\gamma_{*}(t) \\
b\left(u\left(t, \gamma_{*}(t)+\right)\right)=B_{*}(t)
\end{array}\right.
$$

on $[0, T]$ with $B_{*}(t)=B(t, w(t)), \gamma_{*}(t)=\gamma(t)$ and initial datum $u_{0}$;

(2) $w$ solves

$$
\left\{\begin{array}{l}
\dot{w}=F_{*}(t, w), \quad t \in \mathbb{R}^{+}, \\
w(0)=w_{0}
\end{array}\right.
$$

on $[0, T]$ with $F_{*}(t)=F(t, u(t, \gamma(t)+), w)$ a.e.

(3) $\gamma(t)=x_{0}+\int_{0}^{t} \Pi(w(\tau)) d \tau$ for a.e. $t \in[0, T]$.

The following result holds; for a proof see [21].

Theorem 4.2. Let (f), (g), (П), (NC), (b), (F) and (B) hold. Assume that $b(\hat{u})=$ $B(0, \hat{w})$. Then, there exist positive numbers $\delta, \Delta, L, T_{\delta}$, domains $\hat{\mathbb{D}}_{t}$ (for $\left.t \in\left[0, T_{\delta}\right]\right)$ and maps $\widehat{P}\left(t, t_{0}\right): \widehat{D}_{t_{0}} \rightarrow \widehat{D}_{t_{0}+t}\left(t_{0}, t_{0}+t \in\left[0, T_{\delta}\right]\right)$ such that 
(1) $\left(U_{\delta} \times B_{\delta}(\hat{w}) \times(\hat{x}-\delta, \hat{x}+\delta)\right) \subseteq \widehat{D}_{t} \subseteq\left(U_{\Delta} \times B_{\Delta}(\hat{w}) \times(\hat{x}-\Delta, \hat{x}+\Delta)\right)$;

(2) for all $t_{0}, t_{1}, t_{2}$ with $t_{0} \in\left[0, T_{\delta}\right), t_{1} \in\left[0, T_{\delta}-t_{0}\right)$ and $t_{2} \in\left[0, T-t_{0}-t_{1}\right]$, then $\widehat{P}\left(t_{2}, t_{0}+t_{1}\right) \circ \widehat{P}\left(t_{1}, t_{0}\right)=\hat{P}\left(t_{1}+t_{2}, t_{0}\right)$ and $\hat{P}\left(0, t_{0}\right)=\mathrm{Id}$;

(3) for $t_{0} \in\left[0, T_{\delta}\right), t \in\left[0, T_{\delta}-t_{0}\right]$, and $(u, w, x),(\bar{u}, \bar{w}, \bar{x}) \in \widehat{D}_{t_{0}}$

$$
\begin{aligned}
& \left\|\hat{P}\left(t, t_{0}\right)(u, w, x)-\hat{P}\left(t, t_{0}\right)(\bar{u}, \bar{w}, \bar{x})\right\|_{L^{1} \times \mathbb{R}^{m} \times \mathbb{R}} \\
& \quad \leq L\left(\|u-\bar{u}\|_{L^{1}}+\|w-\bar{w}\|_{\mathbb{R}^{m}}+|x-\bar{x}|\right) ;
\end{aligned}
$$

(4) for all $\left(u_{0}, w_{0}, x_{0}\right) \in \widehat{D}_{0}$, the map $t \rightarrow \widehat{P}(t, 0)\left(u_{0}, w_{0}, x_{0}\right)$, defined for $t \in\left[0, T_{\delta}\right]$, solves (32) in the sense of Definition 4.1.

We consider here several examples of applications. Only in Examples 4.4 and 4.8 the boundary is indeed a moving boundary.

Example 4.3 (The Piston Problem 1). Consider a rectilinear tube filled with fluid to the right of a piston. In the isentropic (or isothermal) case this system can be described, using Lagrangian coordinates, by the $p$-system coupled with an ordinary differential equation governing the piston. More precisely

$$
\left\{\begin{array}{l}
\partial_{t} \tau-\partial_{x} v=0, \\
\partial_{t} v+\partial_{x} p(\tau)=0, \\
V=v(t, 0+), \\
\dot{V}=\alpha \cdot(P(t)-p(\tau(t, 0+))),
\end{array}\right.
$$

where $t$ is time, $x$ the Lagrangian mass coordinate, $\tau$ the specific volume, $v$ the Lagrangian speed of the flow, $p$ the pressure in the fluid, $V$ the speed of the piston, $P(t)$ the (given) pressure to the left of the piston and $\alpha$ is the ratio between the section of the tube and the mass of the piston. This problem has been widely considered in the literature, but mainly with pistons having assigned movements, see for instance [112], [113] or [98], § 99. Here, on the contrary, the acceleration of the piston is due to the difference between the pressure of the fluid on its right and that of the outer environment on its left. The problem (33) can be written in the form (32) by putting

$$
\begin{gathered}
u=\left(\begin{array}{l}
\tau \\
v
\end{array}\right), \quad f(u)=\left(\begin{array}{c}
-v, \\
p(\tau)
\end{array}\right), \quad g(u)=0, \\
b(u)=v, \quad w=V, \quad B(t, w)=w \\
F(t, u, w)=\alpha(P(t)-p(\tau)), \quad \Pi(w)=0 .
\end{gathered}
$$

Under suitable assumptions, Theorem 4.2 applies to this case; see also [20]. 
Example 4.4 (The Piston Problem 2). As in Example 4.3, consider a rectilinear and horizontal tube filled with fluid to the right of a piston. In the isentropic (or isothermal) case this system can be described using the Eulerian description of the $p$-system, which leads to the following system:

$$
\left\{\begin{array}{l}
\partial_{t} \rho+\partial_{x} q=0 \\
\partial_{t} q+\partial_{x}\left(\frac{q^{2}}{\rho}+p(\rho)\right)=0 \\
V(t)=\frac{q(t, \gamma(t)+)}{\rho(t, \gamma(t)+)} \\
\dot{V}=\beta\left(p_{\mathrm{ext}}(t)-p(\rho(t, \gamma(t)+))\right), \\
\dot{\gamma}(t)=V(t)
\end{array}\right.
$$

Here, $\rho$ denotes the gas density, $q$ its linear momentum, $p_{\mathrm{ext}}$ a given external pressure, and $\beta$ is the ratio between the section of the tube and the mass of the piston. The problem (34) can be written in the form (32) by putting

$$
\begin{gathered}
u=\left(\begin{array}{l}
\rho \\
q
\end{array}\right), \quad f(u)=\left(\begin{array}{c}
q \\
\frac{q^{2}}{\rho}+p(\rho)
\end{array}\right), \quad g(u)=0, \\
b(u)=\frac{q}{\rho}, \quad w=V, \quad B(t, w)=w, \\
F(t, u, w)=\beta\left(p_{\mathrm{ext}}(t)-p(\rho)\right), \quad \Pi(w)=w .
\end{gathered}
$$

Under suitable assumptions, Theorem 4.2 applies to this case; see also [20].

Example 4.5 (Sewer system with a manhole). Consider a single junction in a sewer network. At $x=0$, a junction joins $k$ horizontal pipes to one vertical manhole. All pipes start at the junction, so that each of them is referred to an abscissa $x \in \mathbb{R}^{+}$. The flow in the $i$-th tube, for $i=1, \ldots, n$, can be described by the Saint-Venant equations [61], see also [54] or formula (108.1) in [98],

$$
\left\{\begin{array}{l}
\partial_{t} A_{i}+\partial_{x} Q_{i}=0 \\
\partial_{t} Q_{i}+\partial_{x}\left(\frac{Q_{i}^{2}}{A_{i}}+p_{i}\left(A_{i}\right)\right)=0
\end{array}\right.
$$

ensuring the conservation of mass and momentum. $A_{i}$ is the wet cross sectional area, $Q_{i}$ the flow in the $x$ direction and $p_{i}$ is a function representing the hydrostatic pressure. As boundary condition, we require the equality of all the hydraulic heads at the junction, that is,

$$
\frac{1}{2 g} \frac{Q_{i_{1}}(t, 0+)^{2}}{A_{i_{1}}(t, 0+)^{2}}+h_{i_{1}}\left(A_{i_{1}}(t, 0+)\right)=\frac{1}{2 g} \frac{Q_{i_{2}}(t, 0+)^{2}}{A_{i_{2}}(t, 0+)^{2}}+h_{i_{2}}\left(A_{i_{2}}(t, 0+)\right)
$$


for all $t \geq 0$ and all $i_{1}, i_{2} \in\{1, \ldots, k\}$, where $h_{i}\left(A_{i}\right)$ is the height of water in the $i$-th tube and $g$ is the gravitational acceleration. Therefore we denote by $\hat{h}(t)$ the value $\frac{1}{2 g} \frac{Q_{i}(t, 0+)^{2}}{A_{i}(t, 0+)^{2}}+h_{i}\left(A_{i}(t, 0+)\right)$ which indeed does not depend on $i$.

The conservation of mass at the junction is expressed by

$$
Q_{M}\left(\hat{h}(t), h_{M}(t)\right)+\sum_{i=1}^{k} Q_{i}(t, 0+)=0,
$$

where $Q_{M}\left(\hat{h}, h_{M}\right)$ is the flow into the manhole. The level $h_{M}$, which is the height of the water inside the storage, changes according to

$$
\dot{h}_{M}(t)=\frac{Q_{M}\left(\hat{h}(t), h_{M}(t)\right)+Q_{\mathrm{ext}}(t)}{A_{M}} .
$$

Here, $A_{M}$ is the horizontal cross section of the manhole and $Q_{\text {ext }}(t)$ is a given external inflow into the manhole. Finally, energy conservation gives

$$
Q_{M}\left(\hat{h}(t), h_{M}(t)\right)=\operatorname{sgn}\left(\hat{h}(t)-h_{M}(t)\right) A_{M} \sqrt{2 g\left|\hat{h}(t)-h_{M}(t)\right|} .
$$

We are thus lead to study the system

$$
\left\{\begin{array}{l}
\partial_{t} A_{i}+\partial_{x} Q_{i}=0, \quad i=1, \ldots, n, \\
\partial_{t} Q_{i}+\partial_{x}\left(\frac{Q_{i}^{2}}{A_{i}}+p_{i}\left(A_{i}\right)\right)=0, \quad i=1, \ldots, n, \\
\hat{h}(t)=\frac{1}{2 g} \frac{Q_{i}(t, 0+)^{2}}{A_{i}(t, 0+)^{2}}+h_{i}\left(A_{i}(t, 0+)\right), \quad i=1, \ldots, n, \\
h_{M}(t)=-\frac{1}{2 g} A_{M}^{2}\left(\sum_{i=1}^{k} Q_{i}(t, 0+)\right)\left|\sum_{i=1}^{k} Q_{i}(t, 0+)\right|+\hat{h}(t), \\
\dot{h}_{M}(t)=\frac{1}{A_{M}}\left(Q_{\mathrm{ext}}(t)-\sum_{i=1}^{k} Q_{i}(t, 0+)\right),
\end{array}\right.
$$

which falls within the class (32) under the following positions:

$$
\begin{gathered}
u=\left(\begin{array}{c}
A_{1} \\
Q_{1} \\
\vdots \\
A_{k} \\
Q_{k}
\end{array}\right), \quad f(u)=\left(\begin{array}{c}
Q_{1} \\
\frac{Q_{1}^{2}}{A_{1}}+p_{1}\left(A_{1}\right) \\
\vdots \\
Q_{k} \\
\frac{Q_{k}^{2}}{A_{k}}+p_{k}\left(A_{k}\right)
\end{array}\right), \\
w=h_{M}, \quad F(t, u, w)=\operatorname{sgn}\left(\hat{h}-h_{M}\right) \sqrt{2 g\left|\hat{h}-h_{M}\right|}+\frac{1}{A_{M}} Q_{\mathrm{ext}}(t),
\end{gathered}
$$




$$
B(t, w)=\left(\begin{array}{c}
0 \\
\vdots \\
0 \\
h_{M}
\end{array}\right), \quad b(u)=\left(\begin{array}{c}
\frac{1}{2 g} \frac{Q_{2}^{2}}{A_{2}^{2}}+h_{2}\left(A_{2}\right)-\hat{h} \\
\vdots \\
\frac{1}{2 g} \frac{Q_{k}^{2}}{A_{k}^{2}}+h_{k}\left(A_{k}\right)-\hat{h} \\
-\frac{1}{2 g A_{M}^{2}}\left(\sum_{i=1}^{k} Q_{i}\right)\left|\sum_{i=1}^{k} Q_{i}\right|+\hat{h}
\end{array}\right) .
$$

Example 4.6 (Supply chains). We now consider a single node of a network of supply chains, connecting $n$ suppliers. Assume we have $\ell$ incoming suppliers and $n-\ell$ outgoing ones. Each outgoing supplier is composed out of a processor and a queue in front of it. For the incoming ones we consider only a processor. The work done by a processor is modeled by

$$
\partial_{t} \rho_{i}(t, x)+\partial_{x}\left(v_{i} \rho_{i}(t, x)\right)=0, \quad x \in \mathbb{R}^{+}, t \in \mathbb{R}^{+}, i=1, \ldots, n,
$$

where $\rho_{i}$ is the density of goods in the $i$-th processor and $v_{i}$ is the (constant) processing velocity. To guarantee the correct orientation of flow, we have $v_{j}<0$ for $j=1, \ldots, \ell$ and $v_{k}>0$ for $k=\ell+1, \ldots, n$, respectively. For the load $q_{k}$ of goods stored in the $k$-th queue, we impose the conservation of mass:

$$
\dot{q}_{k}(t)=f_{k}^{\text {in }}(t)-f_{k}^{\text {out }}\left(q_{k}\right), \quad k=\ell+1, \ldots, n,
$$

where $f_{k}^{\text {in }}$ is the inflow and $f_{k}^{\text {out }}$ is the outflow from the queue to the $k$-th processor. The distribution matrix $A(t)=\left(a_{j k}(t)\right)$, for $j=1, \ldots, \ell$ and $k=\ell+1, \ldots, n$, assigns the percentage $a_{j k}$ of the goods exiting processor $j$ and lining up into the $k$-th queue. Thus, the inflow $f_{k}^{\text {in }}$ is given by

$$
f_{k}^{\text {in }}(t)=\sum_{j=1}^{\ell} a_{j k}(t) v_{j} \rho_{j}(t, 0), \quad k=\ell+1, \ldots, n .
$$

For the outflow we use the relaxed formulation, as presented in [5],

$$
f_{k}^{\text {out }}\left(q_{k}\right)=\min \left(\frac{q_{k}(t)}{\epsilon}, \mu_{k}\right), \quad k=\ell+1, \ldots, n,
$$

with the relaxation parameter $\epsilon>0$ small and $\mu_{k}$ the maximal capacity of the $k$-th processor. Existence and uniqueness for the non-relaxed case were shown in [87], by 
directly using wave front tracking. Then this problem is of type (32), by the positions

$$
\begin{aligned}
& u=\left(\begin{array}{c}
\rho_{1} \\
\vdots \\
\rho_{n}
\end{array}\right), f(u)=\left(\begin{array}{c}
v_{1} \rho_{1} \\
\vdots \\
v_{n} \rho_{n}
\end{array}\right), \\
& w=\left(\begin{array}{c}
q_{\ell+1} \\
\vdots \\
q_{n}
\end{array}\right), F(t, u, w)=\left(\begin{array}{c}
\sum_{j=1}^{\ell} a_{j \ell+1}(t) v_{j} \rho_{j}-\min \left\{\frac{1}{\epsilon} q_{\ell+1}, \mu_{\ell+1}\right\} \\
\vdots \\
\sum_{j=1}^{\ell} a_{j n}(t) v_{j} \rho_{j}-\min \left\{\frac{1}{\epsilon} q_{n}, \mu_{n}\right\}
\end{array}\right), \\
& b(u)=\left(\begin{array}{c}
\min \left\{\frac{1}{\epsilon} q_{\ell+1}, \mu_{\ell+1}\right\} \\
\vdots \\
\min \left\{\frac{1}{\epsilon} q_{n}, \mu_{n}\right\}
\end{array}\right) .
\end{aligned}
$$

Example 4.7 (Blood flow). Following [64] (formulæ (2.3), (2.12), (2.14)), Section 2 of [1], [67] and [34], we consider the 1D model for blood flowing through an artery (29), coupled with a OD (lumped parameter) model describing the averaged mass and flow rate in a given terminal compartment of the circulatory system (e.g., capillary bed, venous circulation), see also [116] and the references therein. The former is modeled by a 1D system of balance laws, as discussed in Section 3.6, while the latter is a system of ordinary differential equations for the mean pressure and flow rate over the whole compartment:

$$
\left\{\begin{array}{l}
\partial_{t} a+\partial_{x} q=0 \\
\partial_{t} q+\partial_{x}\left(\alpha \frac{q^{2}}{a}+\frac{1}{\rho} \pi(a)\right)=-2 \frac{\alpha}{\alpha-1} v \frac{q}{a}, \\
a(t, 0+)=a_{0}\left(1+\frac{p-p_{\mathrm{ref}}}{\beta}\right)^{2} \\
\dot{P}=-\frac{1}{C} Q+\frac{1}{C} q(t, 0) \\
\dot{Q}=-\frac{R}{L} Q+\frac{1}{L} P-\frac{1}{L} P(t, l) .
\end{array}\right.
$$

Here, we used the following notation:
$t$ time
$x \quad$ length along the artery
$a$ arterial cross-section
$\alpha \quad$ Coriolis coefficient
$\pi \quad=\int_{a_{0}}^{a} \tilde{a} p^{\prime}(\tilde{a}) d \tilde{a}$
$q$ arterial flow rate
$a_{0}$ reference cross-section
$\rho$ blood density
$\beta \quad$ arterial wall elasticity
$v$ viscosity coefficient
$P \quad$ compartmental mean
$p=p(a) \quad$ arterial blood pressure
$Q$ compartmental mean flow rate
$C$ compartmental capacitance
$L$ compartmental inductance
$R$ compartmental resistance
$P(t, l) \quad$ outlet compartmental pressure 
From (28) one can see that $\beta=(h E) /\left(\left(1-\sigma^{2}\right) \sqrt{a_{0}}\right)$ and $p(a)=p_{\text {ref }}+\beta\left(\sqrt{a / a_{0}}-1\right)$. Problem (35) is of type (32) with the positions

$$
\begin{gathered}
u=\left(\begin{array}{l}
a \\
q
\end{array}\right), \quad f(u)=\left(\begin{array}{c}
q \\
\alpha\left(\frac{q^{2}}{a}+\frac{1}{\rho} \pi(a)\right)
\end{array}\right), \\
w=\left(\begin{array}{l}
P \\
Q
\end{array}\right), \quad F(t, u, w)=\left(\begin{array}{c}
-\frac{1}{C} Q+\frac{1}{C} q \\
\frac{1}{L} P-\frac{R}{L} Q+\frac{1}{L} \Pi(t)
\end{array}\right), \\
b(u)=a, \quad g(u)=-2 \frac{\alpha}{\alpha-1} v \frac{q}{a}, \quad B(t, w)=a_{0}\left(1+\frac{p-p_{\text {ref }}}{\beta}\right)^{2} .
\end{gathered}
$$

Example 4.8 (Gas-particle interaction). We consider the following model describing the evolution of a solid body inside a compressible fluid:

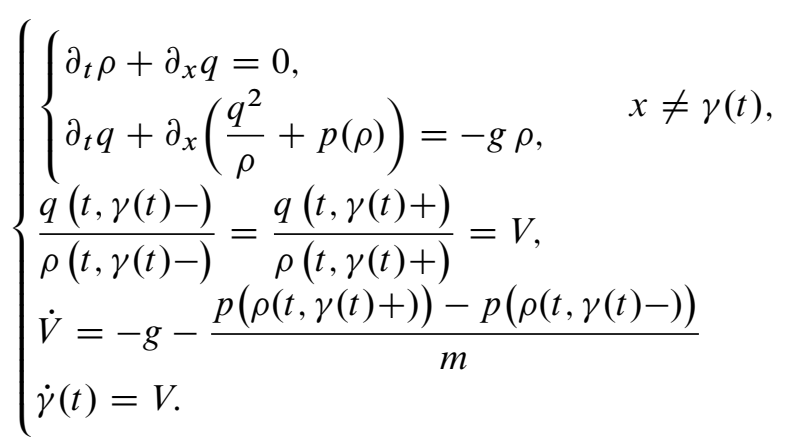

The space variable $x$ is a vertical coordinate oriented upwards; $\rho$ and $q$ are the fluid mass and linear momentum density above and below the particle; $p=p(\rho)$ is the pressure law; $V$ is the speed of the particle sited in $\gamma(t)$ and $m$ is its mass; $g$ is gravity. A justification of the speed law for $\dot{V}$ in (36) is provided by the conservation of energy. System (36) can not be written exactly in the form (32), since here we are considering a copy of the $p$-system also for $x<\gamma(t)$. However existence results for (36) can be obtained with similar techniques; see [22].

4.2. Coupling of traffic models at boundary. We present here a coupling at the boundary between two macroscopic models of traffic, namely the Lighthill-WhithamRichards model and a phase transition model, both of them are based on partial differential equations. The literature contains some other couplings between different traffic models. Among them we recall: the coupling between the follow the leader and Aw-Rascle [99] and the coupling between the follow the leader and a phase transition model [74]. The follow the leader model consists of ordinary differential equations; hence the last two are examples of ODE-PDE coupling. 
Let us consider the following system:

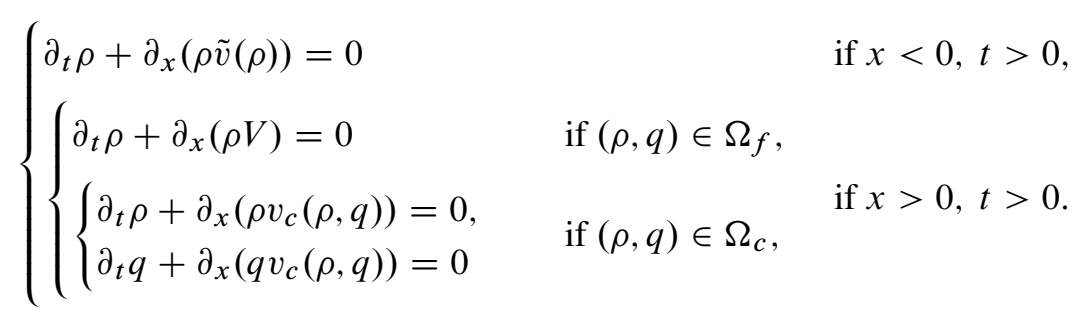

Here the evolution of car traffic is described, for $x<0$, by the Lighthill-WhithamRichards model ([109], [117]), while, for $x>0$, by the phase transition model, introduced in [19]. In the LWR model, the relevant quantities are the density $\rho$ and the velocity $\tilde{v}$, which is a given function depending only on $\rho$. In the phase transition model, $\Omega_{f}$ and $\Omega_{c}$ denote respectively the free and congested phase, $\rho$ is the density of cars, $q$ is a "momentum", $V$ is the maximum speed and $v_{c}$ is the velocity of cars in the congested phase $\Omega_{c}$.

The coupling conditions at $x=0$ between the two models are the conservation of the number of vehicles passing through $x=0$, which reads as

$$
\rho_{l}^{b} V_{\max }\left(1-\frac{\rho_{l}^{b}}{R}\right)= \begin{cases}V \rho_{r}^{b} & \text { if }\left(\rho_{r}^{b}, q_{r}^{b}\right) \in \Omega_{f}, \\ \frac{V \sigma}{R-\sigma} \rho_{r}^{b}\left(\frac{R}{\rho_{r}^{b}}-1\right)\left(1+q_{r}^{b}\right) & \text { if }\left(\rho_{r}^{b}, q_{r}^{b}\right) \in \Omega_{c},\end{cases}
$$

and the maximization of the flux through $x=0$. Under suitable assumption on the model, the following result holds. The complete set of assumptions and the proof are given in [73].

Theorem 4.9. Fix $\rho_{l} \in \mathrm{BV}((-\infty, 0) ;[0, R])$ and $\left(\rho_{r}, q_{r}\right) \in \mathrm{BV}\left((0,+\infty) ; \Omega_{f} \cup \Omega_{c}\right)$. Then there exists $\left(\hat{\rho}_{l},\left(\hat{\rho}_{r}, \hat{q}_{r}\right)\right)$, a weak solution to (37), such that

(1) $\hat{\rho}_{l}(0, x)=\rho_{l}(x)$ for a.e. $x<0$;

(2) $\left(\hat{\rho}_{r}(0, x), \hat{q}_{r}(0, x)\right)=\left(\rho_{r}(x), q_{r}(x)\right)$ for a.e. $x>0$;

(3) for a.e. $t>0$, the trace at $x=0$ of the solution respects the coupling conditions.

Remark 4.10. One can also consider the symmetric situation in which the phase transition model is used in $x<0$ and the LWR model is used in $x>0$. The coupling conditions can be defined in a similar way and an analogous of Theorem 4.9 holds; see [73].

\section{Control and optimization}

Different control and optimization questions, depending on the applications, arise on networks. These can be divided among several groups leading to different analytical 
and numerical techniques. Here we present three different control problems, solved with different techniques. The first one deals with the existence of Nash equilibria for traffic flow on networks in the case of different groups of drivers. The second one considers an optimization procedure of the traffic flow through the junctions. Finally, in the last example, a gas pipeline is considered. Some other important applications, like systems of open canals, production lines, water contamination detection, routing in Internet traffic, are not described in this survey.

5.1. Nash equilibria. In this section we take a somewhat different point of view, regarding traffic flow as the outcome of a decision problem. We assume that each individual driver has a cost $\varphi\left(\tau^{d}\right)$ for early departure and an additional cost $\psi\left(\tau^{a}\right)$ for late arrival. On a general network of roads, the arrival time $\tau^{a}$ is determined by (i) the departure time $\tau^{d}$, (ii) the route taken to reach the destination, and (iii) the overall traffic pattern, which of course depends globally on the decisions of all other drivers. The objective of minimizing the total $\operatorname{cost} \varphi\left(\tau^{d}\right)+\psi\left(\tau^{a}\right)$ leads to two distinct mathematical problems.

(P1) Global optimization problem. Find departure times and routes to destinations in order to minimize the sum of all costs to all drivers.

(P2) Nash equilibrium problem. Find departure times and routes to destinations in such a way that no driver can lower his own cost $\varphi\left(\tau^{d}\right)+\varphi\left(\tau^{a}\right)$ by changing his departure time or switching to a different route.

Note that (P1) is relevant in the case of a central planner who can decide the departure time and the route of every car. On the other hand, (P2) models the more realistic situation where each driver is free to choose his own departure time and route, in order to minimize his own personal cost. The existence, uniqueness, and characterization of solutions to the above problems has been recently studied in [26], [27], [29] in the case of a single road, and in [28] for a network of roads. We survey here the main results.

Let $B_{1}, \ldots, B_{m}$ be the nodes of the network, and call $\gamma_{i j}$ the arc connecting $B_{i}$ with $B_{j}$. The flow of cars along $\gamma_{i j}$ is described by the conservation law

$$
\rho_{t}+\left[\rho v_{i j}(\rho)\right]_{x}=0 \text {. }
$$

Here $t$ is time and $x \in\left[0, L_{i j}\right]$ is the space variable along the arc $\gamma_{i j}$. By $\rho=\rho(t, x)$ we denote the traffic density, while the map $\rho \mapsto v_{i j}(\rho)$ is the speed of cars as function of the density. We assume that $v_{i j}$ is a continuous non-increasing function. If $v_{i j}(0)>0$ we say that the arc $\gamma_{i j}$ is viable. If the two nodes $i, j$ are not directly linked by a road, we simply take $v_{i j} \equiv 0$, so that the arc is not viable. The conservation laws (38) are supplemented by suitable boundary conditions at points of junctions, which will be discussed later. 
We consider $n$ groups of drivers traveling on the network, having different departure and arrival nodes, or different cost functions. For $k \in\{1, \ldots, n\}$, we let $G_{k}$ be the total number of drivers in the $k$-th group. All these drivers depart from a node $B_{d(k)}$ and arrive at a node $B_{a(k)}$, but can choose different paths to reach their destination. Of course, it is assumed that there exists at least one chain of viable arcs

$$
\Gamma \doteq\left(\gamma_{i(0), i(1)}, \gamma_{i(1), i(2)}, \ldots, \gamma_{i(v-1), i(v)}\right)
$$

with $i(0)=d(k)$ and $i(v)=a(k)$, connecting the departure node $B_{d(k)}$ with the arrival node $B_{a(k)}$. We shall denote by

$$
\mathcal{V} \doteq\left\{\Gamma_{1}, \Gamma_{2}, \ldots, \Gamma_{N}\right\}
$$

the set of all viable paths (i.e. concatenations of viable arcs) which do not contain any closed loop. For a given $k \in\{1, \ldots, n\}$, let $\mathcal{V}_{k} \subset \mathcal{V}$ be the set of all viable paths for the $k$-drivers, connecting $B_{d(k)}$ with $B_{a(k)}$. By $U_{k, p}(t)$ we denote the total number of drivers of the $k$-th group, traveling along the viable path $\Gamma_{p}$, who have departed before time $t$.

Definition 5.1. A departure distribution function $t \mapsto U_{k, p}(t)$ is a bounded, nondecreasing, left-continuous function, such that

$$
U_{k, p}(-\infty) \doteq \lim _{t \rightarrow-\infty} U_{k, p}(t)=0
$$

Given group sizes $G_{1}, \ldots, G_{n} \geq 0$, we say that a set of departure distribution functions $\left\{U_{k, p}\right\}$ is admissible if it satisfies the constraints

$$
\sum_{p} U_{k, p}(+\infty)=G_{k}, \quad k=1, \ldots, n .
$$

Since $G_{k}$ is the total number of drivers in the $k$-th group, the admissibility condition (39) means that, sooner or later, every driver of each group has to depart. If the function $U_{k, p}$ is absolutely continuous, its derivative will be denoted by

$$
\bar{u}_{k, p}(t)=\frac{d}{d t} U_{k, p}(t)
$$

Clearly, $\bar{u}_{k, p}$ measures the rate of departures of $k$-drivers traveling along $\Gamma_{p}$.

The overall traffic pattern can be determined by (i) the departure distribution functions $U_{k, p}(\cdot)$, (ii) the conservation laws (38) describing the traffic density on each road, and (iii) a suitable set of conditions at junctions. The departure and arrival costs for drivers of the $k$-th group will be denoted by $\varphi_{k}(\cdot), \psi_{k}(\cdot)$, respectively.

Definition 5.2. An admissible family $\left\{U_{k, p}\right\}$ of departure distributions is globally optimal if it minimizes the sum of all costs to all drivers. 
Definition 5.3. An admissible family $\left\{U_{k, p}\right\}$ of departure distributions is a Nash equilibrium solution if no driver of any group can lower his own total cost by changing departure time or switching to a different path to reach destination.

In other words, calling $A_{p}(t)$ the arrival time of a driver who starts at time $t$ and travels along the path $\Gamma_{p}$, the family $\left\{U_{k, p}\right\}$ is globally optimal if it minimizes the Stieltjes integral

$$
J \doteq \sum_{k, p} \int\left[\varphi_{k}(t)+\psi_{k}\left(A_{p}(t)\right)\right] d U_{k, p}(t)
$$

among all admissible departure distributions.

On the other hand, if $\left\{U_{k, p}\right\}$ is a Nash equilibrium solution, then all drivers in the same group must bear the same total cost. In particular, there must be constants $c_{1}, \ldots, c_{n}$ such that:

(i) For every $t$ in the support of the measure $d U_{k, p}$, one has

$$
\varphi_{k}(t)+\psi_{k}\left(A_{p}(t)\right)=c_{k} .
$$

(ii) For all $t \in \mathbb{R}$, one has

$$
\varphi_{k}(t)+\psi_{k}\left(A_{p}(t)\right) \geq c_{k} .
$$

We emphasize that the arrival times $A_{p}(t)$ heavily depend on the overall traffic conditions, i.e. on all the departure rates $U_{k, p}$ in a global, highly nonlinear way. A first result on the existence of a global optimum and of an equilibrium solution on networks was proved in [28], under the following assumptions.

(A1) For every viable arc $\gamma_{i j}$, the flux function $\rho \mapsto F_{i j}(\rho)=\rho v_{i j}(\rho)$ is continuous, concave down, and non-negative on some interval $\left[0, \bar{\rho}_{i j}\right]$, with $F_{i j}(0)=$ $F_{i j}\left(\bar{\rho}_{i j}\right)=0$. We shall denote by $\left.\left.\rho_{i j}^{*} \in\right] 0, \bar{\rho}_{i j}\right)$ the unique value such that

$$
F\left(\rho_{i j}^{*}\right)=F_{i j}^{\max } \doteq \max _{\rho \in\left[0, \bar{\rho}_{i j}\right]} F_{i j}(\rho), \quad F_{i j}^{\prime}(\rho)>0
$$

for every $\rho \in\left[0, \rho_{i j}^{*}\right)$.

(A2) For each $k \in\{1, \ldots, n\}$, the cost functions $\varphi_{k}, \psi_{k}$ are continuously differentiable and satisfy

$$
\varphi_{k}^{\prime}(t)<0, \quad \psi_{k}^{\prime}(t)>0, \quad \lim _{|t| \rightarrow \infty}\left(\varphi_{k}(t)+\psi_{k}(t)\right)=+\infty .
$$

(A3) Drivers arriving at a node $B_{i}$ from all incoming roads, and who wish to travel along the arc $\gamma_{i j}$, are placed in a buffer (of unlimited capacity) at the entrance of this outgoing arc. Their place in the queue is determined by the time at which they arrive at $B_{i}$, first-come, first-serve. If the queue has nonzero length, then the flux at the entrance of the arc $\gamma_{i j}$ is maximum possible: $F_{i, j}(t, 0+)=F_{i j}^{\max }$. 
Remark 5.4. According to (A2), the cost for early departure is strictly decreasing in time, while cost for late arrival is strictly increasing. The assumption that these costs tend to infinity as $t \rightarrow \pm \infty$ coincides with common sense and guarantees that in a globally optimal as well as in an equilibrium solution the departure rates are compactly supported.

Remark 5.5. In the engineering literature (see for example [68]) it is common to consider a cost of the form $D(t)+\Psi\left(t+D(t)-T_{A}\right)$, defined as the sum of the total duration of the trip $D(t)$ plus a penalty $\Psi$ if the arrival time does not coincide with the target time $T_{A}$. Calling $\tau^{a}(t)=t+D(t)$ the arrival time of a driver departing at time $t$, this cost can be written in the form

$$
\begin{aligned}
D(t)+\Psi\left(t+D(t)-T_{A}\right) & =-t+\left[\tau^{a}(t)+\Psi\left(\tau^{a}(t)-T_{A}\right)\right] \\
& =\varphi(t)+\psi\left(\tau^{a}(t)\right),
\end{aligned}
$$

with $\varphi(t) \doteq-t, \psi(\tau) \doteq \tau+\Psi\left(\tau-T_{A}\right)$.

Remark 5.6. The assumption (A3) greatly simplifies the dynamics at each node $B_{i}$. With reference to Figure 3, by (A3) the traffic density on any outgoing arc $\gamma_{i j}$ can never affect the flow along any incoming arc $\gamma_{k i}$. In particular this implies that, along

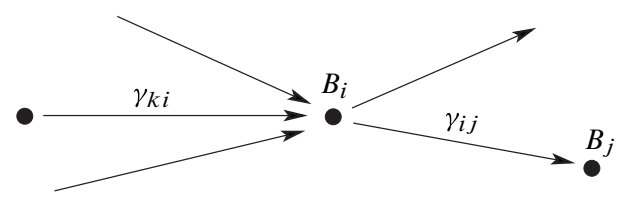

Figure 3. A generic node of the network.

every arc $\gamma_{i j}$, the density satisfies $\rho \in\left[0, \rho_{i j}^{*}\right]$ and the characteristic speed remains positive:

$$
F_{i j}^{\prime}(\rho)=v_{i j}(\rho)+\rho v_{i j}^{\prime}(\rho) \geq 0 .
$$

Notice that this assumption rules out phenomena such as a "spill-back", where the congestion of an outgoing road $\gamma_{i j}$ produces a queue propagating backwards along an incoming road $\gamma_{k i}$.

In the above setting, the main results proved in [28] are as follows.

Theorem 5.7 (Existence of a globally optimal solution). Let the flux functions $F_{i j}$, the cost functions $\varphi_{k}, \psi_{k}$, and the dynamics at intersections satisfy the assumptions (A1)-(A3). Then, for any given population sizes $G_{1}, \ldots, G_{n}>0$, there exists an admissible set of departure distributions $\left\{U_{k, p}\right\}$ providing a globally optimal solution to the traffic flow problem.

Theorem 5.8 (Existence of a Nash equilibrium solution). Let the assumptions (A1)-(A3) hold. 
(i) For any given population sizes $G_{1}, \ldots, G_{n}>0$, there exists at least one admissible family of departure distributions $\left\{U_{k, p}\right\}$ which yields a Nash equilibrium solution.

(ii) In any Nash equilibrium solution, all departure rates $\bar{u}_{k, p}=\frac{d}{d t} U_{k, p}$ are uniformly bounded and have compact support.

Following the direct method of the Calculus of Variations, a globally optimal solution is constructed in [28] by taking the limit of a minimizing sequence $\left\{U_{k, p}^{(v)}\right\}_{\nu \geq 1}$ of admissible departure distributions. The existence of the limit is guaranteed by the "tightness" of the sequence of approximating measures. Namely, for each $\epsilon>0$ there exists $T>0$ (independent of $n$ ) such that the total number of drivers departing at times $t \notin[-T, T]$ is lower than $\varepsilon$.

In the much simpler case where the network reduces to a single road and all drivers have the same departure and arrival cost, it was proved in [26] that the globally optimal solution and the equilibrium solution are unique. Here the departure distribution $U(\cdot)$ for the Nash equilibrium can be obtained as the unique pointwise supremum of a family of admissible distributions, satisfying an additional constraint. The continuous dependence of this equilibrium solution was studied in [29], where the flux function or the cost functions $\varphi, \psi$ were varied.

On the other hand, the existence result stated in Theorem 5.8 for a network of roads is proved by a fixed point argument. By its nature, this topological technique cannot yield information about uniqueness or continuous dependence of the Nash equilibrium.

5.2. Instantaneous control of traffic flow networks. Many existing papers regarding traffic flow on a road network use a (Markov) distribution matrix to distribute traffic flow at intersections [71], [88], [101], [41]. In applications the values of this distribution matrix are not fixed a priori but subject to optimization [86], [69], [80]. In practice, this corresponds to dynamic deviation suggestions for drivers. We discuss the case of feedback controls obtained using instantaneous control. This technique has first been applied in computational fluid dynamics [94], [93]. Here, we survey some results from [85], [80] in a setting of a single traffic intersection with three connected roads $j=1$ and $j=2,3$. We assume that the road $j=1$ is incoming and the roads $j=2,3$ are outgoing. The incoming road is parameterized by $(-\infty, 0]$ and the outgoing ones by $[0, \infty)$, respectively, the node being at $x=0$. We consider, on the road $j$, the Lighthill-Whitham-Richards model [109]:

$$
\partial_{t} \rho_{j}+\partial_{x} f_{j}\left(\rho_{j}\right)=0, \quad f_{j}(\rho)=\rho v_{j}(\rho)
$$

The coupling condition imposed at $x=0$ include the time-dependent distribution Markov matrix $A_{J}(t):=(\alpha(t), 1-\alpha(t))^{\top}$. The conservation of mass through the 
vertex is therefore given by

$$
\left(\begin{array}{l}
f_{2}\left(\rho_{2}(t, 0)\right) \\
f_{3}\left(\rho_{3}(t, 0)\right)
\end{array}\right)=A_{J}(t) f_{1}\left(\rho_{1}(t, 0)\right)
$$

As detailed in the previous section, additional conditions at node $J$ are required in order to obtain unique boundary values for the density $\rho_{j}(t, 0)$, see also [40], [71], [88]. In the case of three connected arcs, the coupling conditions can be solved explicitly and we refer to [85] for more details. Equations (40), (41) have to be supplemented by initial conditions on each road $j$. In the control context the function $t \rightarrow \alpha(t)$ is subject to optimization. Denote by $\rho \rightarrow F_{j}(\rho)$ a non-negative, convex cost functional measuring the density of cars on road $j$. Also, assume that there is a desired distribution parameter $\alpha^{*}$. For a weighting parameter $\delta>0$ we optimize for $A_{J}(t)$ to minimize the following cost functional:

$$
\begin{aligned}
& \frac{1}{2} \int_{-\infty}^{0} \int_{0}^{\infty} F_{1}\left(\rho_{1}(t, x)\right) d t d x+\frac{1}{2} \sum_{j=2}^{3} \int_{0}^{\infty} \int_{0}^{\infty} F_{j}\left(\rho_{j}(t, x)\right) d t d x \\
& \quad+\frac{\delta}{2} \int_{0}^{\infty}\left(\alpha(t)-\alpha^{*}\right)^{2} d t .
\end{aligned}
$$

However, instead of solving an optimal control problem we apply an instantaneous control (IC) concept. The IC proceeds by an implicit Euler semi-discretization in time applied to equations (40), (41). Denote by $t_{k}$ the $k$-th time-step of fixed length $\tau$. The control $\alpha(t)$ is assumed to be piecewise constant on each interval $\left(t_{k}, t_{k+1}\right]$, i.e.,

$$
\alpha(t)=\sum_{k} \alpha_{k+1} \chi_{\left(t_{k}, t_{k+1}\right]}(t) .
$$

The control value $\alpha_{k+1}$ is obtained by solving the problem (42) for given values $\rho_{j}^{k}(x)$ on each road $j$ and given prior control $\alpha_{k}$. Denote by $H_{k+1}$ a discretization of the cost functional (42) on $\left[t_{k}, t_{k+1}\right]$ by the trapezoidal rule:

$$
\begin{aligned}
H_{k+1}= & \frac{\delta}{4}\left(\left(\alpha_{k+1}-\alpha^{*}\right)^{2}+\left(\alpha_{k}-\alpha^{*}\right)^{2}\right)+\frac{1}{4} \int_{-\infty}^{0} F_{1}\left(\rho_{1}^{k+1}(x)\right)+F_{1}\left(\rho_{1}^{k}(x)\right) d x \\
& +\frac{1}{4} \sum_{j=2}^{3} \int_{0}^{\infty} F_{j}\left(\rho_{j}^{k+1}(x)\right)+F_{j}\left(\rho_{j}^{k}(x)\right) d x
\end{aligned}
$$

Problem (43) is an approximation on a single time interval and its solution is only a suboptimal solution compared with the full optimal control problem for (42). 
However, problem (44) can be solved explicitly:

$$
\begin{gathered}
\min H_{k+1} \text { subject to } 0 \leq \alpha_{k+1} \leq 1, \quad A_{J}\left(t^{k+1}\right)=\left(\alpha^{k+1}, 1-\alpha^{k+1}\right), \\
\frac{\rho_{j}^{k+1}(x)-\rho_{j}^{k}(x)}{\tau}+f_{j}\left(\rho_{j}^{k+1}(x)\right)_{x}=0, \\
\left(\begin{array}{l}
f_{2}\left(\rho_{2}^{k+1}(0)\right) \\
f_{3}\left(\rho_{3}^{k+1}(0)\right)
\end{array}\right)=A_{J}\left(t^{k+1}\right) f_{1}\left(\rho_{1}^{k+1}(0)\right) .
\end{gathered}
$$

Note that the minimum to problem (44) (if it exists) only depends on the state of the system $\left(\rho_{j}^{k}(x)\right)_{j}$ at time $t_{k}$ and therefore $\alpha_{k+1}$ is indeed a closed loop control. The value of $\alpha_{k+1}$ may be obtained as solution to the first-order optimality system (45) to problem (44). We denote by $\mathcal{P}_{I}$ the projection to the set $I$. The first-order optimality conditions are given by (45) to (47). The functions $p_{j}(x)$ are obtained as solution to equations (47) and (48). Then, the first-order optimality condition is given by equation (45).

$$
\begin{aligned}
& \mathcal{P}_{[0,1]}\left(\frac{\delta \tau}{2}\left(\alpha^{k+1}-\alpha^{*}\right)+f_{1}\left(\rho_{1}^{k+1}(0)\right)\left(p_{2}(0)-p_{3}(0)\right)\right)=0, \\
& \frac{p_{j}}{\tau}-f_{j}^{\prime}\left(\rho_{j}^{k+1}(x)\right) \partial_{x} p_{j}=-\frac{\tau}{2} F_{j}^{\prime}\left(\rho_{j}^{k+1}(x)\right), \\
& p_{1}(0)=\alpha_{k+1} p_{2}(0)+\left(1-\alpha_{k+1}\right) p_{3}(0) .
\end{aligned}
$$

Theorem 5.9. Assume $F_{j}$ are convex, $f_{j}$ concave and at least $C^{1}$ in their respective arguments. If $\partial_{x} p_{j}(x)$ is non-negative for $j=1, \ldots, 3$, then the function $H_{k+1}$ is convex in $\alpha_{k+1}$.

The proof follows from Theorem 4.1 in [85]. The computed control $\alpha_{k+1}$ is suboptimal compared with a solution to problem (48):

$$
\begin{aligned}
\min _{0 \leq \alpha(t) \leq 1} & \frac{1}{2} \int_{0}^{T}\left(\alpha(t)-\alpha^{*}\right)^{2} d t+\int_{0}^{T} \int_{-\infty}^{0} F_{1}\left(\rho_{1}(t, x)\right) d x d t \\
& +\sum_{j=2}^{3} \int_{0}^{T} \int_{0}^{\infty} F_{j}\left(\rho_{j}(t, x)\right) d x d t
\end{aligned}
$$

subject to (40) and (41).

So far, for networks no rigorous results on problem (48) exist. The main difficulty in the theoretical analysis of (48) is the non-differentiability in $L^{1}$ of the semi-group $\delta_{t} \rho_{j}$ generated by the conservation law (40), see, e.g., Example 1 of [31]. A calculus for the first-order variations of $S_{t} \rho_{j}$ with respect to initial conditions $\rho_{0}$ has been established in Theorems 2.2 and 2.3 of [31] for general 1-D systems of conservation laws with a piecewise Lipschitz continuous $\rho_{0}$ that contains finitely many discontinuities. Therein, the concept of generalized first-order tangent vectors has been introduced to 
characterize the evolution of variations with respect to $u_{0}$, see equations (2.16)-(2.18) in [31]. This result has been extended to BV initial data in [25], [17] and lead to the introduction of a differential structure for $\rho_{0} \rightarrow S_{t} \rho_{0}$, called shift-differentiability, see e.g. [17], Definition 5.1. Related to that equations for the generalized cotangent vectors have been introduced for 1-D systems in [32], Proposition 4. These adjoint equations consist of a nonconservative transport equation ([32], equation (4.2)) and an ordinary differential equation ([32], equations (4.3)-(4.5)) for the tangent vector and shift in the positions of possible shocks in $\rho(t, x)$, respectively. Necessary conditions for a general optimal control problem including explicit boundary conditions have been established in Theorem 1 of [32]. However, this result was obtained using strong assumptions on $\rho_{0}$ (see Remark 4 in [32] and Example 5.5 in [17]). Analytical results for optimal control problems in the case of a scalar hyperbolic conservation law with a convex flux have also been developed using a different approach in [122]. For all approaches, the extension of the results to the network case is still open.

5.3. Controllability and optimal control of gas networks . We discuss controllability [81] and optimal control problems for flow in gas networks [45], [90] while similar results are also available in the context of connected open canals, see for example [16], [50], [51], [105], [82], [106], [78], [107], [108]. So far, controllability results exist only in the realm of strong $e^{1}$ solutions leading to strong bounds on initial and desired data. We recall a recent result on controllability of gas networks [81]. Similar results also exist for open canals where the main difference is the source term and the pressure in the momentum equation. In the case of gas networks, results have been established for $p(\rho)=a^{2} \rho$ and a source term modeling pipe wall friction. In the case of open canals, the pressure is $p(h)=\frac{g}{2} h^{2}$ and a friction term due to the slope of the canal is considered. A controllability result for the general $p$-system with general source term is only available if it is a particular case of the general controllability result for quasi-linear systems [105].

To exemplify the ideas we consider a network of two connected pipes with a controllable compressor station located at vertex $J$ with two connected pipes. In the following the pipes are labeled $j=1$ and $j=2$ for the incoming and outgoing pipe, respectively. We also assume that pipe one is parametrized such that $x \in[-L, 0]=$ : $I_{1}$ and pipe two such that $x \in[0, L]=: I_{2}$ with the node being the compressor at $x=0$. In case of $\mathcal{C}^{1}$ solutions the governing equations in quasilinear form on pipes $j \in\{1,2\}$ are

$$
\begin{gathered}
\partial_{t} y_{j}+A^{j}\left(y_{j}\right) \partial_{x} y_{j}=G_{j}\left(t, x, y_{j}\right) \quad \text { in } I_{j} \times[0, T], \\
\Psi\left(y_{1}(t, 0), y_{2}(t, 0)\right)-u(t)=0,
\end{gathered}
$$

and boundary conditions at $x=-L$ and $x=L$. In case of gas networks we have $y=(\rho, q)$ and a friction source term of the type $G(y)=\left(0,-c_{0} \frac{q|q|}{\rho}\right)^{\top}$ for a nonnegative constant $c_{0}$. The flux function is $F(y)=\left(q, \frac{q^{2}}{\rho}+a^{2} \rho\right)^{\top}$ with sound speed 
$a$ and $A^{j}=D_{y} F(y)$. The function $t \rightarrow u(t)$ is the (unknown) control. In the case of a gas network, $u(t) \geq 0$ may correspond to the power applied at a compressor station at time $t$. If supplied with enough power, the compressor increases the pressure downstream in order to balance the friction effects. A similar control is also active in the case of water flow in open canals. Here, $u(t)$ models the opening of underflow gates in connected canal systems. The spill of the underflow gate is used to regulate the water level downstream. The coupling condition in the case of a compressor [45] is given by

$$
\Psi=\left(q_{1}(t, 0)-q_{2}(t, 0),-q_{2}(t, 0)\left({\frac{\rho_{2}(t, 0)^{\kappa}}{\rho_{1}(t, 0)}}^{\kappa}-1\right)\right)
$$

for some $\kappa>0$. Given finite times $T>\widetilde{T}>0$ we consider exact controllability to a desired boundary data $y_{B}(t)$ which should be attained at $x=L$, for $t \geq t^{*}$ and $t^{*} \in(\widetilde{T}, T)$. The initial data $y_{j}(t, 0)$ and boundary data at $x=-L$ are given. Then, the controllability problem is to find a solution $u \in \ell^{1}(0, T)$ such that (49) has a classical solution on $[0, T]$ that satisfies the exact controllability condition $y_{2}(t, L)=y_{B}(t)$ for all $t \in\left[t^{*}, T\right]$.

Theorem 5.10. Assume that $A^{j}$ are smooth functions and the matrices possess two real eigenvalues $\lambda_{i}^{j}$ for $i=1,2$. Let $\widetilde{T}$ be sufficiently large and choose $t^{*} \in(\widetilde{T}, T)$. Assume any solution $y_{j}$ fulfills $\lambda_{1}^{j}\left(y_{j}\right)<0<\lambda_{2}^{j}\left(y_{j}\right)$. Then, there exists a number $\delta>0$ such that for all initial and boundary data of $\mathcal{C}^{1}$-norm lower than $\delta$ and which are additionally $\ell^{1}$-compatible at $x=0$ and all desired data $y_{B} \in \mathcal{C}^{1}(0, T)$ with $\left\|y_{B}\right\| \leq \delta$ the following statement holds true.

There exists a control function $u \in \mathcal{C}^{1}(0, T)$ such that $y_{2}(t, L)=y_{B}(t)$ for all $t \in\left[t^{*}, T\right]$.

The assumption on the sign of the eigenvalues is crucial to all existing controllability results and allows one to determine a priori the outgoing and incoming characteristics at the boundary. The $\ell^{1}$-compatibility condition ensures the existence of a global $\mathcal{C}^{1}$ solution $y_{j}$ in $[0, T] \times I_{j}$. More details are given in Theorem 3.2 of [81]. The result also extends to perturbations of stationary solutions: Consider a subsonic steady-state solution to (49) and corresponding boundary data $y_{B}$. Then, there exists a small $\ell^{1}$ neighborhood of the boundary data and a $\ell^{1}$ control $u$ such that exact controllability holds for all data from the neighborhood ([81], Theorem 3.2). The results extend to tree-like networks having as many compressor stations as desired boundary data. The result also extends to coupled systems of general quasi-linear equations and general nonlinear coupling conditions $\Psi$. The precise assumptions are found in [81], Theorem 2.1. The global exact controllability of quasi-linear hyperbolic systems has been studied also in [108], [82]. Therein, the exact controllability is used to describe the problem to drive the system to a desired state at a given finite time. The results for networks extend those by driving the state at some node to a given desired state and by using nodal controls instead of initial value controls. The simpler case of linear 
coupling conditions $\Psi\left(y_{1}(t, 0), y_{2}(t, 0)\right)=A\left(y_{1}(t, 0), y_{2}(t, 0)\right)$ is studied in [82], [50]. A result for nodal control in the case of tree-like networks has been discussed in [107]. Therein, all conservative variables are controlled independently and the results apply for the Saint-Venant equation only. There is a relation to the exact boundary observability of flows in a tree-like network of open canals [78] where it has been shown that the initial data can be determined by the observation of the boundary data.

Next, we present results on the existence of optimal controls in the presence of discontinuities [46], [45]. We consider equation (49) in conservative form for $y=\left(y_{1}, y_{2}\right)$ with

$$
\partial_{t} y_{j}(t, x)+\partial_{x} F\left(y_{j}\right)=G_{j}\left(t, x, y_{j}\right), \Psi(y(0))-u(t)=0 .
$$

The result for the optimal control problem heavily relies on the analytical properties of the solution. Denote by $\varepsilon_{t}\left(y_{0}, u\right)$ the weak solution to equation (50) for initial data $y_{0}=\left(y_{1,0}, y_{2,0}\right) \in L^{1}$, control $u \in L^{1}$ both of small TV-norm and sufficiently close in $L^{1}$ to a subsonic constant $(\bar{y}, \bar{u})$ such that $\Psi\left(\bar{y}_{1}, \bar{y}_{2}\right)-\bar{u}=0$. It can be proven that $\mathcal{E}$ fulfills the following estimate for all $t \in[0, T]$ :

$$
\left\|\varepsilon_{t}\left(y_{0}, u\right)-\varepsilon_{t}\left(\tilde{y}_{0}, \tilde{u}\right)\right\| \leq L\left(\left\|y_{0}-\tilde{y}_{0}\right\|+\int_{0}^{t}\|u(s)-\tilde{u}(s)\| d s\right) .
$$

This bound immediately yields an existence result for the optimal control $u(\cdot)$ to the nonlinear constrained problem (51).

Theorem 5.11. Assume functions $\mathcal{g}_{0}$ and $\mathcal{F}_{1}$ are non-negative and lower semicontinuous with respect to the $L^{1}$-norm in their respective arguments $u$ and $y=\left(y_{1}, y_{2}\right)$. Then, for some $\delta>0$ and given initial condition $y_{0} \in\left\{\bar{y}+L^{1}\left(\mathbb{R}^{+} ; \mathbb{R}^{2}\right), \operatorname{TV}(y) \leq \delta\right\}$, the problem

$$
\min \mathscr{g}_{0}(u)+\int_{0}^{T} \mathscr{g}_{1}\left(\mathcal{E}_{s}\left(y_{0}, u\right)\right) d s
$$

admits a minimum on

$$
\left\{u \in \bar{u}+L^{1}\left(\mathbb{R}^{+}\right), \operatorname{TV}(u)+\operatorname{TV}\left(y_{0}\right)+\left\|\Psi\left(y_{0}(0+)\right)-u(0+)\right\| \leq \delta\right\} .
$$

The proof follows from Proposition 2.4 of [45]. Theorem 5.11 holds true for general $2 \times 2$ balance laws and we refer to [45] for more details. In particular, the result applies to the control of pumping stations in water networks and underflow gates in open canals, where the dynamics is described by Saint-Venant equations. For a further characterization of the minimum to (51) a first-order optimality system is required. As in the case of traffic flow discussed above, so far no stronger results are available. We refer to the discussion at the end of the previous section for further references. A formal computation of adjoint equations and corresponding coupling conditions has been used in numerical methods and we refer, for example in the case of the Euler equations, to [90]. For the isothermal Euler equations those equations 
have been derived in [90]. For constant initial data the adjoint coupling conditions together with the adjoint equations to (50) are a well-posed problem having a unique solution. No further results in this direction have been proposed so far.

\section{Numerics}

The discussed flow problems on networks are spatially one-dimensional hyperbolic balance laws. There exists a vast literature on possible numerical schemes to discretize system (3) which we do not review at this point due to lack of space.

We first focus on the discretization of the coupling condition (4) in the realm of finite-volume schemes. For a general introduction to finite volume schemes we refer for example to the textbooks [97], [104].

We then consider multi-dimensional models, which can be used to deduce coupling conditions for the one-dimensional reductions. Specifically examples are provided for car traffic and gas pipes.

6.1. Coupling conditions for finite volume schemes. The equations (3) are approximated numerically using a finite volume method on a regular grid of cell size $x_{i+1}-x_{i}=\Delta x$ and time step $t^{m+1}-t^{m}=\Delta t$, chosen so that the CFL condition [52] $\lambda_{\max } \Delta t \leq \Delta x$ is satisfied, where $\lambda_{\max }$ is the largest wave speed. The discretization is done for each component $u_{j}$ separately. In a finite volume method the cell average $\bar{U}_{j, i}^{m}$ of $u_{j}$ in cell $i$ at time $t^{m}$ is given by

$$
\bar{U}_{j, i}^{m}:=\frac{1}{\Delta x} \int_{x_{i-\frac{1}{2}}}^{x_{i+\frac{1}{2}}} u_{j}\left(x, t^{m}\right) d x .
$$

The evolution of the cell average over time $\Delta t$ is

$$
\bar{U}_{j, i}^{m+1}=\bar{U}_{j, i}^{m}-\frac{\Delta t}{\Delta x}\left(\left(\mathscr{F}_{j}\right)_{i+\frac{1}{2}}^{m}-\left(\mathcal{F}_{j}\right)_{i-\frac{1}{2}}^{m}\right)+\bar{G}_{j, i}^{m},
$$

where in Godunov's method [75] $\left(\mathcal{F}_{j}\right)_{i+\frac{1}{2}}^{m}=\mathscr{F}_{j}\left(\bar{U}_{j, i}^{m}, \bar{U}_{j, i+1}^{m}\right)$ denotes the numerical flux for $u_{j}(t, x)$ through the boundary between cells $i$ and $i+1$ and where $\bar{G}_{j, i}^{n}$ is an approximation to $\frac{1}{\Delta x} \int_{t^{m}}^{t^{m+1}} \int_{x_{i-\frac{1}{2}}}^{x_{i+\frac{1}{2}}} g_{j}\left(t, x, u_{j}(x, t)\right) d x d t$ obtained by a suitable quadrature rule. In Godunov's method the exact solution to a Riemann problem posed at the cell boundary $i+\frac{1}{2}$ is used to define the numerical flux $\left(\mathcal{F}_{j}\right)_{i+\frac{1}{2}}^{m}$. Then in Godunov's method the numerical approximation to $u_{j}(t, x)$ is obtained by the piecewise constant reconstruction

$$
u_{j}(t, x)=\sum_{i} \sum_{m} \bar{U}_{j, i}^{m} \chi_{\left[x_{i-\frac{1}{2}}, x_{i+\frac{1}{2}}\right] \times\left[t^{m}, t^{m+1}\right]}(t, x) .
$$


The coupling condition (4) at the node induces a boundary condition for equation (4). Numerically, a construction of boundary values can be provided under the assumptions on $\bar{u}_{j, 0}$ given by Theorem 2.13 and condition (11) on $\Psi$. To fix the notation we assume that at time $t^{m}$ the cell averages in the first cell $i=0$ of the connected arcs (parameterized by $x>0$ ) are given by $\bar{U}_{j, 0}^{m}$ for $j=1, \ldots, n$ and that they are sufficiently close to $\bar{u}_{j, 0}$ such that condition (11) holds true. Denote by $\sigma \rightarrow \mathscr{L}_{\kappa}\left(u_{0}, \sigma\right)$ the $\kappa$-th Lax curve through the state $u_{0}$ for $\kappa=1,2$. Then, using Newton's method we solve for $\left(\sigma_{1}^{*}, \ldots, \sigma_{n}^{*}\right)$ the nonlinear system

$$
\Psi\left(\mathscr{L}_{2}\left(\bar{U}_{1,0}^{m}, \sigma_{1}\right), \ldots, \mathscr{L}_{n}\left(\bar{U}_{n, 0}^{m}, \sigma_{n}\right)\right)=0
$$

A unique solution exists due to (11). The boundary value $\bar{U}_{j, 0}^{m+1}$ at time $t^{m+1}$ is then given by equation (52) for $i=0$ and with

$$
\bar{U}_{j,-1}^{m}:=\mathscr{L}_{2}\left(\bar{U}_{j, 0}^{m}, \sigma_{j}^{*}\right)
$$

Obvious modifications of this construction allow one to treat also incoming arcs (parameterized by $x<0$ ) and coupling conditions including ordinary differential equations or controls. The construction yields a first-order approximation to the coupling condition. So far, no higher order discretizations of coupling conditions have been proposed in the literature.

6.2. Multi-dimensional approximation of coupling conditions. In many flow models on networks the correct coupling condition connecting the dynamics on different arcs is not known in full detail. However, its precise form is crucial from many point of views: it determines the applicability of the model, as well as its theoretical properties and the numerical implementation. In this section we present two ideas to at least numerically derive and validate coupling conditions for flow models. Here, the node is not considered as single point but as a multi-dimensional domain. We exemplify the ideas by studying an example in traffic flow [86] and the Euler equations of gas dynamics [84]. Other examples are found in the literature [91], [92]. Unfortunately, so far no theory which rigorously allows one to derive coupling conditions from multi-dimensional flow descriptions exists.

The idea presented in [84] is to use a numerical study of an extended twodimensional model for a T-shaped intersection to investigate proposed coupling conditions. A theoretical discussion of conditions in the one-dimensional case has been carried out for the Euler equations in [47]. The resolution at the node is achieved by introducing a local zooming of the situation, see for example Figure 4. This yields a two-dimensional domain representing the T-shaped intersection fitting with corresponding initial flow conditions in each arc. The governing equations in the zoomed 

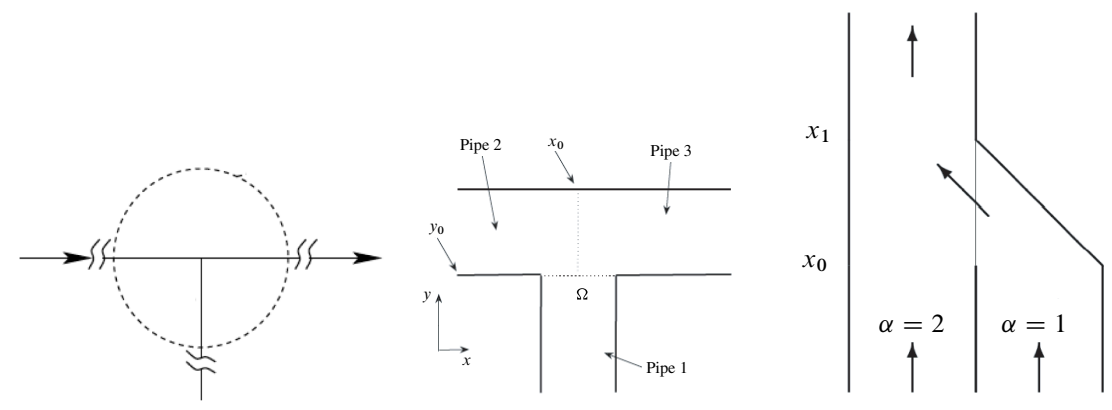

Figure 4. Multi-dimensional approximation for coupling conditions at nodes in the case of traffic flow (right) and gas dynamics (middle). The generic one-dimensional situation is depicted at the left. The multi-dimensional approximations are used to obtain nodal conditions for the generic situation.

region are given by the polytropic Euler system:

$$
\left\{\begin{aligned}
\partial_{t} \rho+\partial_{x}(\rho u)+\partial_{y}(\rho v) & =0 \\
\partial_{t}(\rho u)+\partial_{x}\left(u^{2} \rho+p\right)+\partial_{y}(\rho u v) & =0 \\
\partial_{t}(\rho v)+\partial_{x}(\rho u v)+\partial_{y}\left(v^{2} \rho+p\right) & =0 \\
\partial_{t} E+\partial_{x}(u(E+p))+\partial_{y}(v(E+p)) & =0 \\
E=\frac{p}{\gamma-1}+\frac{1}{2} \rho\left(u^{2}+v^{2}\right) &
\end{aligned}\right.
$$

We now assign initial data according to the connected arcs, see (57) for the example depicted in Figure 4. We compute a fully evolved solution using a high-order finitevolume numerical scheme and average on subdomains. The resulting values are then compared with the predictions of the coupling conditions of the one-dimensional model [47]. This approach is motivated by the following considerations: The onedimensional model is typically used for simulation purposes since it is computationally less expensive than the two-dimensional model, see Table 1. Further, it is assumed that the two-dimensional dynamics happen on a much faster timescale compared to the dynamics in the arcs. Hence, we use the simulation results for the one-dimensional model. We therefore consider only the region close to the center of the computational domain. Furthermore, we know that in the case of constant initial data, the theoretical results predict constant states at the intersection. Hence, we are interested in time-independent values obtained by two-dimensional computations. This motivates the averaging procedures given by equation (58). If one is interested in the transient behavior, the results of the two-dimensional simulations should be used as follows: For a combination of data the tables of the two-dimensional results yield the state which eventually will be attained by the dynamics at the intersection. 
Table 1. CPU times in seconds for a two- and a one-dimensional model on a tree-shaped geometry and three connected pipes, respectively.

\begin{tabular}{ll|ll}
\multicolumn{2}{c|}{$2 \mathrm{D}$} & \multicolumn{2}{c}{$1 \mathrm{D}$} \\
Grid & CPU [sec] & Grid & CPU [sec] \\
\hline $40 \times 40$ & 19.41 & 40 & 0.71 \\
$80 \times 80$ & 172.95 & 80 & 2.79 \\
$120 \times 120$ & 615.51 & 120 & 6.28 \\
$240 \times 240$ & $>15$ min & 240 & 24.14 \\
\hline
\end{tabular}

In more detail, we prescribe initial data in the depicted arcs or pipes one to three by $U=(\rho, \rho u, E)$ as

$$
U(x, y, 0)= \begin{cases}\left(\rho_{1,0}, q_{1,0}, 0, E_{1,0}\right), & (x, y) \in \text { Pipe } 1 \\ \left(\rho_{2,0}, 0, q_{2,0}, E_{2,0}\right), & (x, y) \in \text { Pipe 2 } \\ \left(\rho_{3,0}, q_{3,0}, 0, E_{3,0}\right), & (x, y) \in \text { Pipe 3 }\end{cases}
$$

where $\left(\rho_{i, 0}, q_{i, 0}, E_{i, 0}\right)$ is the constant initial data. The size of the domain in the artificial new variable is $\bar{w}$. We compute at every time step the cell averages $\bar{U}_{i}$ for $\delta \in(0, \infty):$

$$
\bar{U}_{i}(\delta, t):=\frac{1}{\left|\Omega_{i}^{\delta}\right|} \int_{\Omega_{i}^{\delta}} U(x, y, t) d x d y, \quad i=1, \ldots, 3 .
$$

The domains $\Omega_{i}^{\delta}$ are overlapping domains with size $\delta+\bar{w} \times \bar{w}$. They extend in the direction of the arcs with length $\delta$ and overlap in an area of size $\bar{w}^{2}$. For a given tolerance $\epsilon$ we simulate the two-dimensional model (56) and (57) until time $\tau$ such that

$$
\sum_{i=1}^{3} \partial_{t} \sup _{\gamma}\left\|\partial_{\delta} \bar{U}_{i}(\tau, \gamma)\right\|_{2} \leq \epsilon
$$

The termination criterion is a measure for the current dynamics inside each subdomain and indicates changes in the averaged moments. The criterion proposed implies that only the traces of the solution near the intersection is important to the comparison. Exiting waves are lost to the comparison. In the one-dimensional model we observe waves moving out of the intersection leaving boundary values $\widetilde{U}_{i}$ independent of time behind. Hence, for sufficiently small $\delta$ we average on domains $\Omega_{i}^{\delta}$ which are close to the center of our computational domain and consider only traces of the two-dimensional solution. The measure (59) indicates whether or not we reached stationary values for the averaged moments. Once we satisfied the termination crite- 
rion we denote by $U_{i}(\tau, 0)$ the reference values for comparison with the theoretical predictions, see also [84].

We show that even in the case of single intersection, a full two-dimensional simulation is computationally expensive. We compare the computational times for the twodimensional simulation on a T-shaped intersection geometry with a one-dimensional simulation on three connected pipes until time $T=0.5$ using a second-oder finite volume scheme. Nodes with three connected arcs are most common when considering applications in gas pipeline systems. A detailed report where the final states obtained by the coupling conditions proposed in [47] as well as the averaged values of the numerical simulation is given in [84]. In general, the complex two-dimensional dynamics cannot be fully captured by the one-dimensional models and we can only verify if the qualitative behavior coincides. We observe similar trends and the persistence of qualitative features and the order of magnitude of the values is the same. Arcs with higher values for the density, velocity and energy in the theory correspond to those in the numerical simulation. Numerical examples are given in Figure 5.
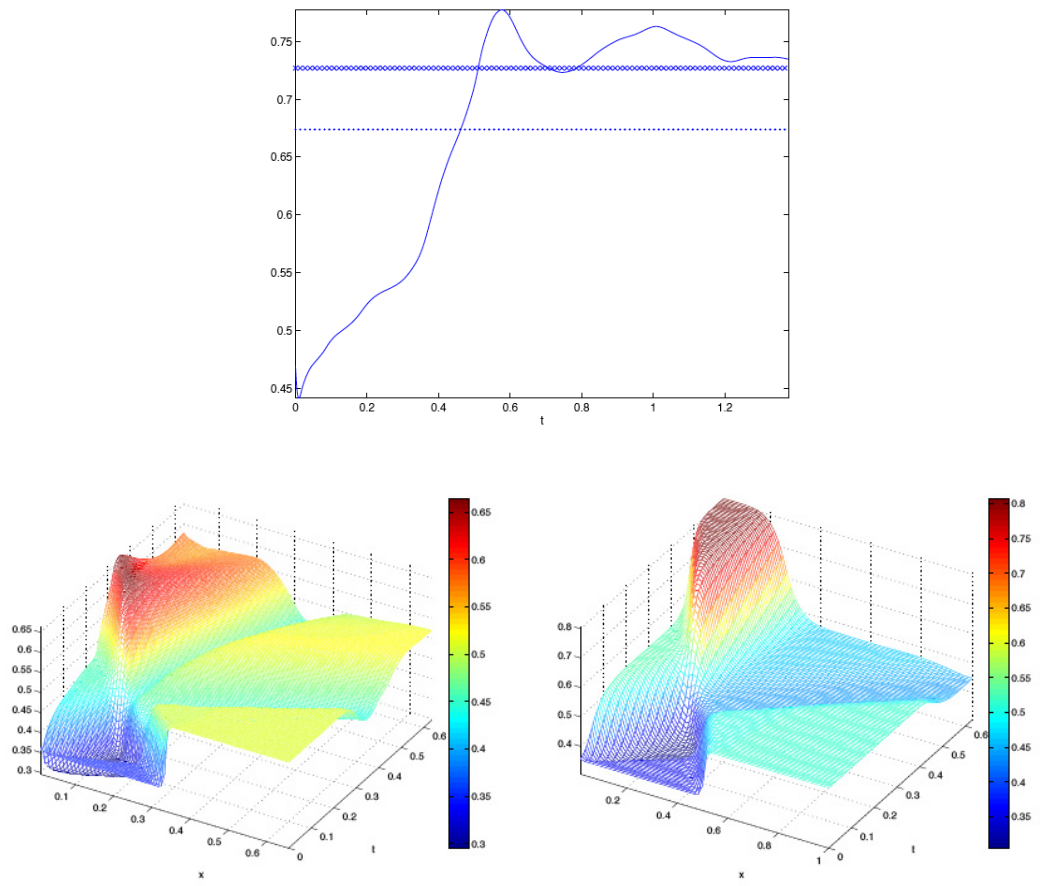

Figure 5. First line: Graph of the numerical solution $\rho(t, \cdot, \cdot)$ obtained by simulation of the twodimensional situation over time. The volume averaged two-dimensional results are indicated by a cross $(\mathrm{X})$. The theoretical predictions are indicated by a dot. Second line: Solution $\rho(t, x)^{2 d}$ and $\rho^{\text {ana }}(t, x)$ in middle and right part of the picture, respectively. The node is located at $x=0$. 
Therein, we report the graph of the densities $\rho^{\text {ana }}(t, x)$ and $\rho^{2 d}(t, x)$ on a single connected arc. For given initial data the multi-dimensional Euler equations with initial data as in (57) is solved and the volume averaged two-dimensional results are shown. We further compute the values obtained from the coupling conditions proposed in [47]. Those are then used to numerically simulate the one-dimensional model. The full two-dimensional solution is averaged along the pipe and shown for comparison. The initial data generates a more complex wave pattern in the twodimensional situation and we therefore cannot expect to have a constant state once all interactions have taken place. However, close to the node also the solution in the multi-dimensional case approaches a constant value at zero.

For traffic flow networks a different procedure has been proposed in [86]. Here, traffic flow on unidirectional roads at a motorway junction is described by a number of roads which merge or disperse. A numerical simulation of a model with multiple roads and considering a long-time limit leads to comparable coupling conditions in traffic flow. To exemplify the procedure and the results consider the situation of Figure 4. The Aw-Rascle-Zhang [8], [124] approach to the full multi-lane equations with $N$ roads labeled by $\alpha$ and with density $\rho_{\alpha}(t, x)$ and velocity $u_{\alpha}(t, x)$ on lane $\alpha$ are

$$
\begin{aligned}
& \partial_{t} \rho_{\alpha}+\partial_{x}\left(\rho_{\alpha} u_{\alpha}\right)=\left(\frac{1}{T_{\alpha-1}^{L}} \rho_{\alpha-1}-\frac{1}{T_{\alpha}^{R}} \rho_{\alpha}\right)\left(1-\delta_{\alpha, 1}\right) \\
&+\left(\frac{1}{T_{\alpha+1}^{R}} \rho_{\alpha+1}-\frac{1}{T_{\alpha}^{L}} \rho_{\alpha}\right)\left(1-\delta_{\alpha, N}\right), \\
& \partial_{t}\left(\rho_{\alpha} u_{\alpha}\right)+\partial_{x}\left(\rho_{\alpha} u_{\alpha} w_{\alpha}\right)=\left(\frac{1}{T_{\alpha-1}^{L}} u_{\alpha-1} \rho_{\alpha-1}-\frac{1}{T_{\alpha}^{R}} u_{\alpha} \rho_{\alpha}\right)\left(1-\delta_{\alpha, 1}\right) \\
&+\left(\frac{1}{T_{\alpha+1}^{R}} u_{\alpha+1} \rho_{\alpha+1}-\frac{1}{T_{\alpha}^{L}} u_{\alpha} \rho_{\alpha}\right)\left(1-\delta_{\alpha, N}\right), \\
& w_{\alpha}=u_{\alpha}+p\left(\rho_{\alpha}\right) .
\end{aligned}
$$

We refer to [8] for more details on the function $p$ and theoretical properties of the conservation law. The terms $T_{\alpha}^{L, R}$ are the lane changing rates from lane $\alpha$ to the left or to the right. For $u_{\alpha}=U\left(\rho_{\alpha}\right)$, the equations simplify to a scalar multi-lane traffic flow model:

$$
\begin{aligned}
\partial_{t} \rho_{\alpha}+\partial_{x}\left(U\left(\rho_{\alpha}\right) \rho_{\alpha}\right)= & \left(\frac{1}{T_{\alpha-1}^{L}} \rho_{\alpha-1}-\frac{1}{T_{\alpha}^{R}} \rho_{\alpha}\right)\left(1-\delta_{\alpha, 1}\right) \\
& +\left(\frac{1}{T_{\alpha+1}^{R}} \rho_{\alpha+1}-\frac{1}{T_{\alpha}^{L}} \rho_{\alpha}\right)\left(1-\delta_{\alpha, N}\right)
\end{aligned}
$$

In general, a full network flow is accurately described by the multi-lane equations. To save computational time, one restricts the model to a single lane on most part of each arc and, via a local zooming, uses multiple lanes only close to nodes. Consider 
the situation of Figure 4 with a one-dimensonal scalar traffic flow model

$$
\partial_{t} \rho_{i}+\partial_{x} \rho_{i} U\left(\rho_{i}\right)=0
$$

posed on the three connected arcs $i=1,2,3$. For simplicity let the node be located at $x=0$. Coupling conditions for the one-dimensional model and corresponding boundary values $\bar{\rho}(t, 0)$ have been proposed for example in [96], [71]. We model the local traffic by a two-lane situation $(N=2)$ where the first road merges into the second road. The first road is also closed at $x_{1}$. In the area $\left(x_{0}, x_{1}\right)$ cars change only from lane one to two, i.e., $T_{2}^{R}=0$ and $T_{1}^{L}$ is chosen as $1 / T_{1}^{L}=\omega\left(1-\rho_{2}\right) / T^{*}(x)$ where $T^{*}$ is linearly decreasing function for $x \in\left(x_{0}, x_{1}\right)$. The initial conditions in the case of equation (60) are

$$
\rho_{1}(x, 0)=\rho_{1,0} H\left(x-x_{1}\right)+\rho_{2,0} H\left(x_{1}-x\right), \rho_{2}(x, 0)=\rho_{3,0} H\left(x_{1}-x\right) .
$$

The values $\rho_{i, 0}, i=1,2,3$, are the boundary values at time $t$ of the one-dimensional models at the node, i.e., $\rho_{i, 0}=\rho_{i}(t, 0)$. In order to obtain boundary values for equation (61) we assume the region of the node in model (60) is of order $\epsilon \ll 1$. Rescaling the domain and the lane changing rates accordingly leads to $T_{\alpha}^{L, R} \rightarrow$ $\epsilon T_{\alpha}^{L, R}$. In rescaled coordinates $\hat{x}=x / \epsilon$ we look now for the asymptotic values of model (60) at $t, x \rightarrow \infty$. The desired boundary values $\bar{\rho}_{i}(t+\Delta t, 0)$ for (61) for small times $\Delta t$ are then given by

$$
\begin{gathered}
\bar{\rho}_{1}=\rho_{1}(t=\infty, x=-\infty), \quad \bar{\rho}_{2}=\rho_{2}(t=\infty, x=-\infty), \\
\bar{\rho}_{3}=\rho_{2}(t=\infty, x=\infty) .
\end{gathered}
$$

A numerical comparison can be found in [86]. Therein, the multi-lane model has been computed for different (constant) initial conditions and different type of nodes in the case of the Lighthill-Whitham-Richards model. The multi-lane model and the boundary values $\bar{\rho}_{i}$ proposed in [41] give nearly coinciding results if the outgoing roads are initially empty. In other cases the difference in the predicted boundary values can reach up to order one. Comparisons with coupling conditions proposed for the Aw-Rascle model [88], [70] have not yet been obtained. Furthermore, a comparison for a larger multi-lane network with one-dimensional models is still open.

\section{Applications}

We present here two successful applications of the theory and numeric developed in the previous sections. More precisely we first focus on the use of GPS data from mobile sensor to monitor the load of large road networks. Then we describe the theory of metallic frame structures and its application to the case of vascular stents. 
7.1. Traffic monitoring from mobile sensors using GPS data. The use of data to monitor traffic has a long history. Traditional methods to gather information on average flows and velocities were mainly based on static sensors, such as radars, coils etc. More recently cameras were used, mostly to detect flow at highways entrances or other critical points of large networks.

The most impressive revolution in this area is represented by the use of data (mostly given by GPS devices) from mobile sensors. The latter include both the case of smart mobile phones as well as onboard car equipments. More precisely we will briefly describe two main projects: The so called Mobile Millennium Project of the group led by Alex Bayen at the University of California at Berkeley and the traffic monitoring system implemented by Octo Telematics in Italy and various European capitals.

7.1.1. Mobile Century experiment and Mobile Millennium project. The Mobile Century experiment is a prototype data collection system, which was launched on February 8, 2008 and used to estimate traffic conditions for a day on I-880 near San Francisco, CA. The experiments involved 165 UC Berkeley students, 100 vehicles carrying Nokia N95 phones driving repeatedly in loops of six to ten miles in length for eight hours. The highway section was selected because of frequent heavy congestion. The vehicles monitored by the system represented a penetration (number of cars monitored as percentage of the total) of approximately $2 \%$ to $5 \%$. Moreover, data were also collected by existing loop detectors to assess the experiment results. A fairly complete account of the experiment achievements can be found in [83] and a description of the mathematical methods (including flows on networks using PDE models) can be found in [123].

This experiment further evolved in a monitoring system for the San Francisco bay area, called Mobile Millennium, see .

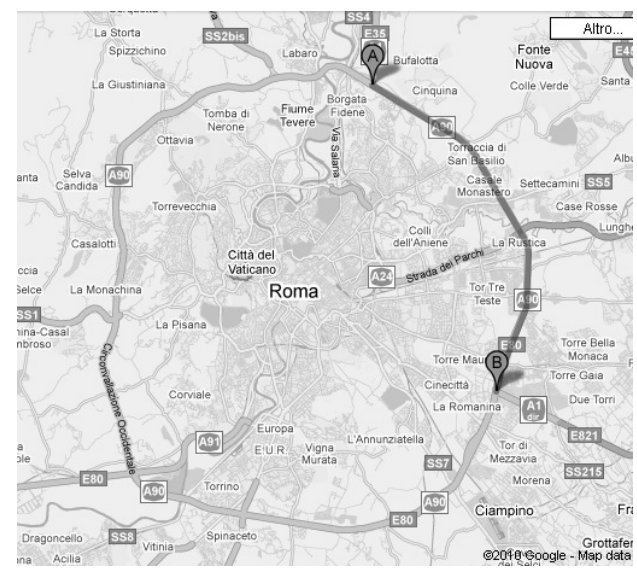

Figure 6. Considered part of the Grande Raccordo Anulare of Rome, Italy (by Google maps). 
7.1.2. Octotelematics and clear boxes. The company Octo Telematics S.p.a.@ (see ) collects data obtained from a special set of sensors mounted on cars, named clear box. Each clear box registers data at some time instants, then sends the information to the base for processing. Final data include position (by a GPS unit) and velocity of the car, as well as registration (measurement) time and processing time. The system is used to monitor traffic on the Italian highway network as well as in a number of European big cities.

An experiment on the use of data from clear boxes in combination with a model based on conservation laws was presented in [53]. Data were collected from cars traveling on the Rome urban area, more precisely on a part of the Grande Raccordo Anulare (GRA) in Rome, Italy. GRA is a circular urban highway with three lanes for each direction, see Figure 6, with speed limit of $130 \mathrm{~km} / \mathrm{h}$. The whole GRA is in general highly congested, and long queues are usual. At the time of the experiments around 750,000 cars (in Italy) were equipped with clear boxes, while now the number exceeds 1.5 million, with penetration rate more than $2 \%$. All data were gathered on June 19, 2009 (Friday), from 6 a.m. to 11 a.m. In Figure 7 we report the space-time positions registered by the sensors for each direction of the GRA. The size of the circles denotes the velocity (small circle stands for small velocity while large circle stands for large velocity).
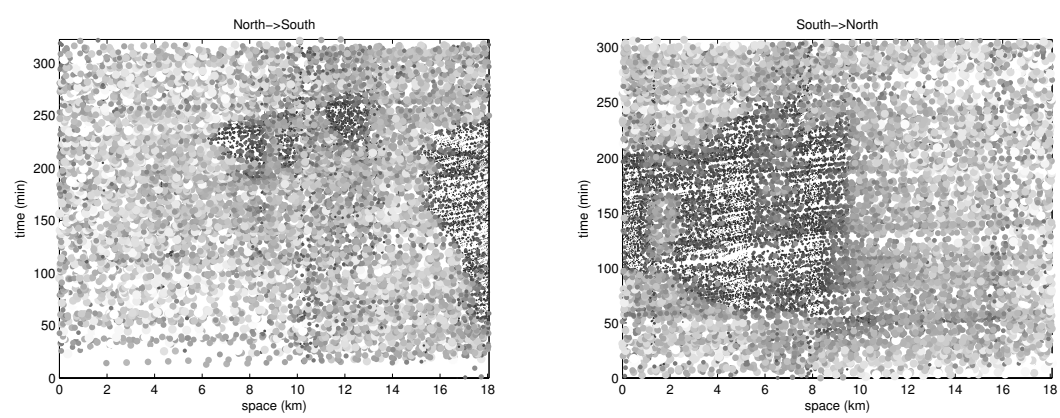

Figure 7. Data plot, North $\rightarrow$ South direction (left) and South $\rightarrow$ North direction (right). The space $(\mathrm{km})$ is reported on the $x$-axis and the time (min) on the $y$-axis. Circle's size is proportional to the velocity of the cars.

7.2. Metallic frame structures (with J. Tambača). Hyperbolic nets, which do not have an a priori association with flows, arise in many applications, such as, for example, bridge structures in civil engineering (see, e.g., [115] and the references therein), cardiovascular applications, design of tissue scaffolds, etc. A prototypical example of a hyperbolic net that will be considered in this section comes from cardiovascular treatment and it models a cylindrical metallic mesh, called stent, that is inserted into a human blood flow conduit (e.g., coronary artery) to counteract disease-induced blood 
flow constriction; see Figure 8. Classical approaches in biomedical engineering liter-

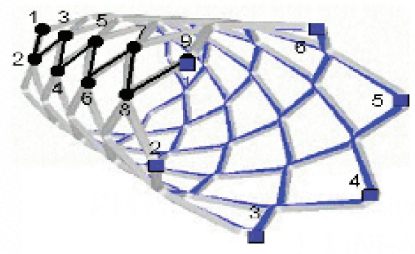

Figure 8. Example of a stent used in coronary angioplasty.

ature model the mechanical properties of stents by approximating a stent as a single 3D elastic body, typically using commercial software packages based on 3D finite element method structure approximations. It is well-known that simulating slender objects such as stent struts using 3D approaches is computationally very expensive, typically producing simulation results with poor accuracy do to insufficient mesh refinement, limited by the large memory requirements. To get around these difficulties, in [121], [38] it was proposed to model stents as hyperbolic nets, approximating the slender stent struts using the theory of $1 \mathrm{D}$ curved rods. The stent mesh is described as a non-directed graph in $\mathbb{R}^{3}$ whose edges correspond to stent struts. This leads to a hyperbolic stent net problem.

To model endovascular stents and, more generally, the entire class of hyperbolic net problems, the following three main steps need to be addressed:

(1) Geometry. Describing how individual components, such as stent struts, comprise a complex global net structure such as stent. This is given by a mathematical description of a stent net domain as a graph, with parameterizations of the graph's edges modeling the middle lines of each stent strut;

(2) Physics. Describing the mechanical properties of each individual component. This is given by a set of mathematical equations describing the physical conservation/balance laws and the constitutive laws satisfied by each component. In the stent application the stent struts will be modeled using the AntmanCosserat curved rod model (63)-(67) [4].

(3) Coupling conditions. Describing the mechanics of contact between the individual components (stent struts). This is given by a set of contact/coupling conditions.

The Antman-Cosserat model for a single curved rod can be written in the form of a system of nonlinear hyperbolic balance laws [4]:

$$
\partial_{t}(\boldsymbol{g}(\boldsymbol{U}))=\partial_{s}(\boldsymbol{h}(\boldsymbol{U}))+\boldsymbol{k}(\boldsymbol{U}), \quad t>0, s \in[0, l],
$$

where $t>0$ corresponds to time, and $s \in[0, l]$ is the spatial coordinate that parameterizes the middle line of the curved rod. Here, $\boldsymbol{g}$ is a (possibly) nonlinear combination 
of the state variables, $\boldsymbol{h}$ is the flux and $\boldsymbol{k}$ the source term. A particular form of these functions can be deduced from system (63)-(67). The middle line of the curved rod at time $t$ is parameterized by $\boldsymbol{r}(s, t)$. The state variable $\boldsymbol{U}=\left(\boldsymbol{d}_{1}, \boldsymbol{d}_{2}, \boldsymbol{d}_{3}, \boldsymbol{u}, \boldsymbol{v}, \boldsymbol{\omega}, \boldsymbol{p}\right)$ contains 21 unknown functions that correspond to the following physical and geometric quantities:

- $\left(\boldsymbol{d}_{1}, \boldsymbol{d}_{2}, \boldsymbol{d}_{3}\right)$ is the local coordinate system defined by the cross-sections of the curved rod. The cross-sections are spanned by the vector functions $\boldsymbol{d}_{1}$ and $\boldsymbol{d}_{2}$, where $\boldsymbol{d}_{3}:=\boldsymbol{d}_{1} \times \boldsymbol{d}_{2}$. Vectors $\left\{\boldsymbol{d}_{1}, \boldsymbol{d}_{2}, \boldsymbol{d}_{3}\right\}$ are orthonormal;

- components $u_{i}$ of $\boldsymbol{u}$ in the local basis $\left\{\boldsymbol{d}_{i}\right\}\left(\boldsymbol{u}=\sum_{i=1}^{3} u_{i} \boldsymbol{d}_{i}\right)$ measure flexure $\left(u_{1}\right.$ and $\left.u_{2}\right)$, and torsion $\left(u_{3}\right)$, where $\boldsymbol{u}$ is defined via $\partial_{s} \boldsymbol{d}_{i}=\boldsymbol{u} \times \boldsymbol{d}_{i}, i=1,2,3$;

- components $v_{i}$ of $\boldsymbol{v}:=\partial_{s} \boldsymbol{r}$ in the local basis $\left\{\boldsymbol{d}_{i}\right\}\left(\boldsymbol{v}=\sum_{i=1}^{3} v_{i} \boldsymbol{d}_{i}\right)$ measure shear $\left(v_{1}\right.$ and $\left.v_{2}\right)$, and dilatation $\left(v_{3}\right)$;

- $\boldsymbol{\omega}$, which is defined via $\partial_{t} \boldsymbol{d}_{i}:=\boldsymbol{\omega} \times \boldsymbol{d}_{i}, i=1,2,3$, is associated with angular velocity of the cross-sections of the curved rod;

- $\boldsymbol{p}:=\partial_{t} \boldsymbol{r}$ describes the velocity of the middle line of the curved rod.

To simplify the presentation of the Antman-Cosserat model we denote by $\boldsymbol{D}$ the matrix consisting of the local coordinate vectors, $\boldsymbol{D}:=\left(\boldsymbol{d}_{1}, \boldsymbol{d}_{2}, \boldsymbol{d}_{3}\right) \in \mathrm{SO}(3)$. The Antman-Cosserat curved rod model can now be described by the following system of equations (see equations (9.2)-(9.3d) on p. 298 in [4]):

$$
\begin{aligned}
\partial_{t} \boldsymbol{d}_{i} & =\boldsymbol{\omega} \times \boldsymbol{d}_{i}, \quad i=1,2,3, \\
\partial_{t} \boldsymbol{u} & =\partial_{s} \boldsymbol{\omega}-\boldsymbol{u} \times \boldsymbol{\omega}, \\
\partial_{t} \boldsymbol{v} & =\partial_{s} \boldsymbol{p}, \\
\partial_{t}\left(\rho \boldsymbol{D} \boldsymbol{J} \boldsymbol{D}^{\top} \boldsymbol{\omega}\right) & =\partial_{s} \boldsymbol{m}+\boldsymbol{v} \times \boldsymbol{n}+\boldsymbol{l}, \\
\rho A \partial_{t} \boldsymbol{p} & =\partial_{s} \boldsymbol{n}+\boldsymbol{f} .
\end{aligned}
$$

Here, $\boldsymbol{m}$ and $\boldsymbol{n}$ are the contact moment and contact force, respectively, while $\boldsymbol{f}$ and $\boldsymbol{l}$ are the outside forcing terms. The constant $A>0$ describes the cross-sectional area, while $\boldsymbol{J}$ is a positive definite, diagonal matrix, containing the information about the geometry of cross-sections.

This system is supplemented with the constitutive relations describing the given material via

$$
\boldsymbol{m}=\boldsymbol{D} \mathrm{m}\left(\boldsymbol{D}^{\top} \boldsymbol{u}, \boldsymbol{D}^{\top} \boldsymbol{v}\right) \quad \text { and } \quad \boldsymbol{n}=\boldsymbol{D n}\left(\boldsymbol{D}^{\top} \boldsymbol{u}, \boldsymbol{D}^{\top} \boldsymbol{v}\right),
$$

where $\mathrm{m}$ and $\mathrm{n}$ are the corresponding constitutive functions. Hyperelastic rods were, for example, considered in [38] (which means that there exists a stored energy function $W$ such that the functions $\mathrm{m}$ and $\mathrm{n}$ can be obtained as the partial derivatives of $\mathcal{W}$ with respect to the first and second variable, respectively). 
The set of three equations appearing in (63) describe the evolution of the crosssection. The second and third vector equations described by (64) and (65) are just the compatibility conditions among the second-order derivatives (e.g., vector equation (65) reads $\boldsymbol{v}_{t}=\boldsymbol{r}_{s t}=\boldsymbol{r}_{t s}=\boldsymbol{p}_{s}$ ). The last two vector equations, namely equations (66) and (67), describe the balance of angular momentum (the angular impulsemomentum law) and the balance of linear momentum (the linear impulse-momentum law), respectively.

Equations (63)-(67) need to be supplemented with initial and boundary conditions. The boundary conditions in our case are the coupling conditions that hold at the vertices where stent struts meet.

Two types of coupling conditions are physically reasonable and consistent with the global weak formulation of the stent problem [39], [18]:

(1) The kinematic coupling conditions which require continuity of velocities at the vertex. More precisely, for each vertex $\mathbf{v}$ the velocities $\boldsymbol{p}_{j}, j=1, \ldots, n$, of the middle lines, and the velocities $\omega_{j}, j=1, \ldots, n$, of the cross-sections of the $n$ rods meeting at $\mathbf{v}$, must satisfy

$$
\omega_{1}=\omega_{j} \quad \text { and } \quad p_{1}=p_{j}, \quad j=1, \ldots, n .
$$

(2) The dynamic coupling conditions which require balance of contact forces and contact moments of all the rods meeting at the same vertex v. More precisely,

$$
\sum_{j=1}^{n} \pm \boldsymbol{m}_{j}=0 \quad \text { and } \quad \sum_{j=1}^{n} \pm \boldsymbol{n}_{j}=0
$$

where the plus or minus sign depends on the parameterization of each branch meeting at the vertex $\mathbf{v}$ (incoming vs. outgoing branches). The solution of the stent net problem is independent of the choice of parameterization of the branches.

One can further show that the eigenvalues of the sub-system (64)-(67) are $\pm \sqrt{\mu_{i}}$ where $\mu_{i}>0$ are the eigenvalues of $\nabla(\mathrm{m}, \mathrm{n})$. Since there is no differentiation with respect to the spatial variable $s$ in the three equations described by (63), the corresponding eigenvalues of the Jacobian matrix of the flux function are all zero. Thus, system (63)-(67) has 21 eigenvalues (characteristic speeds), nine of which as zero, and the remaining 12 eigenvalues come in pairs $\pm \sqrt{\mu_{i}}, i=1, \ldots, 6$, with $\mu_{i}>0$.

7.2.1. Simplified problem: the nonlinear wave system. Mathematical properties of the Antman-Cosserat model are similar to those of the first-order system associated with the nonlinear wave equation: $u_{t t}=\left(f(u) u_{x}\right)_{x}$ :

$$
\begin{aligned}
& u_{t}=w, \\
& v_{t}=w_{x},
\end{aligned}
$$




$$
w_{t}=(f(u) v)_{x}
$$

The corresponding characteristic speeds of system (68)-(70) are given by $\lambda_{1}=$ $0, \lambda_{2,3}= \pm \sqrt{f(u)}$. The first equation does not contain the flux term, just as the equations in (63). The second equation (69) is the compatibility condition among the second-order derivatives which reflects the same condition as the equations (64), (65) in the Antman-Cosserat model. Finally, the third equation (70) incorporates the main physics in the problem, describing the balance of momentum, just as the equations (66) and (67). Specifying the form of $f$ as a function of $u$ determines the physical properties of the nonlinearly elastic string, modeled by (68)-(70).

A net/network problem in which the physics of each edge is modeled by the nonlinear wave equation satisfies the following coupling conditions at vertex $\mathbf{v}$, joining $n$ branches of the net:

(1) Continuity of displacement (which implies continuity of velocity):

$$
u_{1}=u_{j}, \quad j=1, \ldots, n .
$$

(2) Balance of contact forces:

$$
\sum_{j=1}^{n} f\left(u_{j}\right) u_{j_{x}}=0 .
$$

Weak formulation. Suppose, for the moment, that our net consists of one vertex $\mathbf{v}$ joining $n$ branches, each parameterized so that $x=0$ corresponds to $\mathbf{v}$, with $x \in(0, l)$. To get a weak formulation of the net problem, consider $n$ copies of the nonlinear wave equation holding along each branch. Multiply each equation by a test function $\phi_{j} \in H^{1}([0, l]), j=1, \ldots, n$, corresponding to the displacement of the $j-t h$ branch, and integrate by parts with respect to $x$ from 0 to $l$. To obtain a weak formulation of the (global, coupled) net problem, which is defined on the union of $n$ edges meeting at $\mathbf{v}$, take the sum, as $j=1, \ldots, n$. Define the test space for the displacement $u$ of the global net problem to be given by $V=\{\phi=$ $\left.\left(\phi_{1}, \ldots, \phi_{n}\right): \phi_{k} \in H^{1}([0, l]), \phi_{1}(0)=\cdots=\phi_{m}(0)\right\}$. Notice that the continuity of the displacement at vertex $\mathbf{v}$ is included in the definition of the test space via the last set of conditions in $V$. After summing over all the edges meeting at $\mathbf{v}$, one obtains, for all $\phi \in V$,

$$
\begin{aligned}
& \frac{d^{2}}{d t^{2}} \sum_{j=1}^{n} \int_{0}^{l} u_{j} \phi_{j} d x+\sum_{j=1}^{n} \int_{0}^{l} f\left(u_{i}\right) \partial_{x} u_{j} \partial_{x} \phi_{j} d x \\
& \quad=\sum_{j=1}^{n} f\left(u_{j}(l, t)\right) \partial_{x} u_{j}(l, t) \phi_{j}(l)-\sum_{j=1}^{n} f\left(u_{j}(0, t)\right) \partial_{x} u_{j}(0, t) \phi_{j}(0) .
\end{aligned}
$$

After taking into account the kinematic coupling condition in $V$, one can see that the last term in this equation is exactly the left-hand side of the dynamic contact 
condition (2) above, multiplied by $\phi_{i}(0)$ which all have the same value at the vertex. Setting this term equal to zero enforces the dynamic coupling condition. To complete the weak formulation of the problem, we supplement this integral formulation with initial data, and with boundary data at $x=l$. Thus, the weak formulation of the net problem reads: for every $\phi \in V$ we have

$$
\begin{gathered}
\frac{d^{2}}{d t^{2}} \sum_{j=1}^{n} \int_{0}^{l} u_{j} \phi_{j} d x+\sum_{j=1}^{n} \int_{0}^{l} f\left(u_{i}\right) \partial_{x} u_{j} \partial_{x} \phi_{j} d x \\
=\sum_{j=1}^{n} f\left(u_{j}(l, t)\right) \partial_{x} u_{j}(l, t) \phi_{j}(l) .
\end{gathered}
$$

With this approach both the PDE and the coupling conditions are satisfied simultaneously and naturally in the corresponding weak formulation.

Figure 9 shows numerical simulation using a conforming Finite Element Method of a net with 8 vertices forming a cube in 3D. The initial displacement, shown in the first panel on the left, gives rise to two waves, traveling in opposite directions. The two waves interacts with a vertex, giving rise to a reflected and a transmitted wave, while, at the same time, displacing the location of the vertex itself.
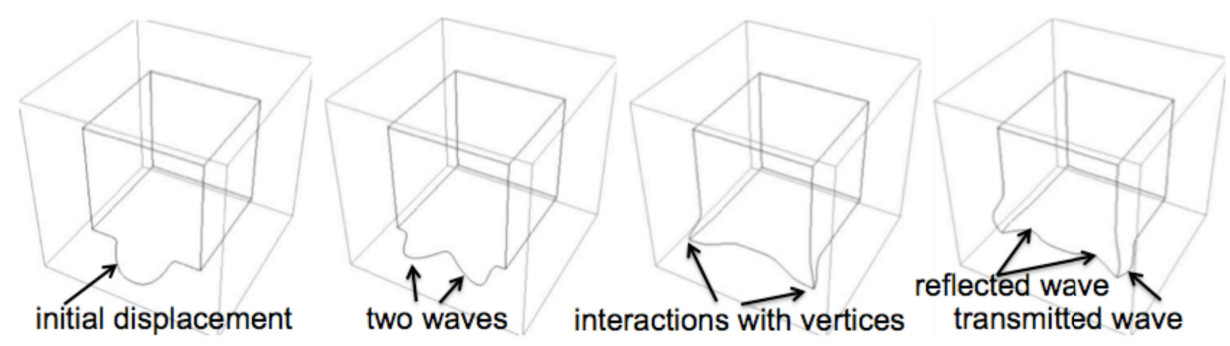

Figure 9. Solution of the nonlinear wave equation $u_{t t}=\left(\left(0.5+u^{2}\right) u_{x}\right)_{x}$ on a net with 8 vertices and 3 edges meeting at each vertex (the cube skeleton). The exterior cube shown in all the figures is not a part of the net, but serves as a reference frame for all the figures. The initial displacement $u$ is shown in the figure on the left, and the initial velocity is $u_{t}=0$. The initial hump breaks into two waves traveling in opposite directions as shown in the second figure from the left, until each wave starts interacting with a vertex. As a result, the vertex is displaced, as shown in the third figure from the left, and the reflected and transmitted waves form, as shown in the fourth figure from the left. This scenario continues along the net.

A complete analysis of this problem is open. The results obtained for this simplified problem will shed light on the structure of solutions to the more complicated stent net problem, and the corresponding class of net problems in general. 


\section{Future perspectives}

Among the many possible extensions of the current state of research on flow on networks, we will only point out some problems of interest. We split the presentation between the different covered topics discussing modeling, theoretical and numerical directions of possible future research.

8.1. Modeling. From the modeling point of view many applications require a largescale network with an interest often not on the detailed dynamics but in global flow properties. There are in general two ways to proceed either by reducing the dynamics or by homogenization of fine-scale dynamics.

A typical approach in deriving simplified models on network models is to start from the full multi-dimensional problem (e.g., a 3D problem), and perform dimension reduction using some clever averaging techniques to obtain a reduced, 1D model, that holds on each branch (edge) of the large-scale 1D hyperbolic network. In nonlinear problems, averaging the multi-dimensional problem gives rise to closure problems. Depending on the application at hand, such a problem can be resolved by either assuming an ad hoc closure (see, e.g., [34]), or by designing a clever dimension reduction approach that gives rise to a reduced problem without an ad hoc closure (see, e.g., [35], [111]). Once a reduced model is obtained, the next question is to impose the "correct" coupling conditions between the different branches (edges). A particular choice for the coupling conditions determines the particular physics of the underlying problem. Different coupling conditions give rise to a different network problems. From the mathematics point of view, it is desirable to prove, using rigorous analysis, that the coupling conditions used in the given network problem are consistent with the full 3D formulation of the problem. This is difficult to prove in general, but there are examples where this can been done. One example is the 1D hyperbolic net problem arising in modeling metallic frame structures such as stents, discussed in 7.2. Starting from 3D elasticity, it was shown in [39], [18] that the two coupling conditions discussed in 7.2 requiring continuity of velocity of displacement, and balance of contact forces at each vertex of the net, are consistent with 3D elasticity. Proving this kind or result for general net and network problems is open, and it depends on the particular application at hand. Therefore, proving that a given 1D hyperbolic network problem is consistent with the original full multi-dimensional problem is generally open.

On the other hand, starting from a given possibly large scale 1D hyperbolic network, it is also of interest to derive a multi-dimensional models to capture global flow properties. This may be called homogenization with respect to the graph structure. Here, instead of resolving the detailed dynamics, the focus is on deriving equations for average flow properties leading possibly to a true multi-dimensional hyperbolic equation for averaged quantities. A procedure to derive such equations has been investigated in [89]. Therein, a 1D linear transport model on arcs and a simple queuing 
dynamics at nodes has been considered. The multi-dimensional hyperbolic equation has been derived using a kinetic equation posed on an extended state space including the graph topology of the network. Mean-field and Chapman-Enskog expansion lead to an equation on multiple spatial dimensions. However, the extension to the general problem including in particular the nonlinear transport discussed in this review is still unsettled.

Models involving 1D hyperbolic nets and networks also arise in multi-physics and multi-scale problems that incorporate different physical phenomena occurring at different spatial and temporal scales. From the computational point of view, using reduced, $1 \mathrm{D}$ hyperbolic net models in a global, multi-physics problem is particularly suitable making the coupled multi-physics problem solvable in a relatively reasonable time frame. Examples include coupling the stent net problem, discussed in Section 7.2, with the motion of an elastic/viscoelastic arterial wall and blood flow [36], coupling the stent net problem with biochemistry and porous medium equations to study drug release into arterial tissue of drug-eluting stents [125], the coupling of the 1D hyperbolic net model describing the mechanical properties of metallic frame buildings with a 3D model of fluid flow describing the swaying of buildings exposed to strong winds, etc. Modular computational schemes are particularly useful for such simulations [33]. However, various analysis questions related to the coupling of the reduced 1D hyperbolic net models with multi-dimensional models in a multi-physics problem are still open. They relate to both the analysis of the underlying computational models as well as to the study of existence, and, more generally, well-posedness of the underlying solutions.

8.2. Theory. From the theoretical point of view the most challenging current problems are probably the control questions for flows on networks. Today, there exist results on the optimal control as well as controllability and stabilization of flows on networks by many authors treating scalar and systems case. From the application point of view also the design of networks (e.g. number and degree of nodes) with dynamics governed by hyperbolic equations is an open problem. For a recent result in the case where the dynamics could be computed explicitly we refer to [103]. However, the general nonlinear case as well as more general coupling conditions are not covered and remain open for future investigations.

Different control problems may be discussed using the Nash equilibria approach. Here, many theoretical questions remain still open. For example, an important open question is whether the results presented in Section 5.1 remain valid if the assumption (A3) in Section 5.1 is replaced by more realistic conditions determining the flow at intersections. For example, it would be of interest to investigate the existence of global optima and Nash equilibrium solutions in connection with the Riemann solvers at junctions proposed in [41], [71]. Another interesting question is the dynamic stability of the equilibrium solution discussed in Section 5.1. Assume that, day after day, each driver can change his own departure time seeking to lower the sum of his 
departure and arrival cost. As a result, the distribution of departures as well as the overall traffic pattern will change each day. This leads to a dynamical system on the space of all departure distributions, having Nash equilibria as steady states. It is natural to expect that, after several days, the departure distribution should converge to a Nash equilibrium. To study this problem, for a single group of drivers traveling on a single road, two specific models were introduced in [29]. Surprisingly, numerical simulations suggest that the unique Nash equilibrium is unstable, while the orbits approach a chaotic attractor. No theoretical analysis has yet proved or disproved this conjecture.

8.3. Numerics. From a numerical point of view the intense research on theoretical properties of conservation laws on networks is not well matched by suitable numerical analysis. In particular, when treating nonlinear source terms it is known that the resolution of stationary states needs special treatment leading to the class of wellbalanced schemes. Also, for large-scale networks high-order numerical schemes may be interesting in order to reduce the number of points required for the resolution of the underlying dynamics on a single arc. The question of a high-order resolution of the coupling condition as well as the extension of well-balanced schemes for networks could be directions of possible future research.

Further, the control results presented are also not yet accompanied by suitable numerical methods for various reasons. Preliminary results on the discretization of Lyapunov functions used for controllability questions have been obtained in [9]. Therein it has been shown that the theoretically expected decay rate is obtained also for a class of finite volume schemes. However, the result is limited to a single arc and the extension to networks still open. Similar results on numerical schemes for solving an optimal control problem on networks are still missing. The development of efficient numerical methods having similar properties as theory suggests would be highly desirable for future applications.

Another direction concerns the development of efficient numerical methods for multi-physics and multi-scale problems. Here, one obstacle is the possibly different time-scales arising. A network model governed by hyperbolic transport is typically solved with an explicit in time scheme. This way the graph structure may be exploited using highly parallel codes. However, if an underlying micro-dynamics is on faster time-scale an implicit in time scheme would be more desirable. This would immediately couple the dynamics on different arcs. The problem to design methods to treat simultaneously coupled problems over various time scales in the case of network flow problems is still unsolved. 


\section{References}

[1] J. Alastruey, K. H. Parker, J. Peiró, and S. J. Sherwin, Lumped parameter outflow models for 1-D blood flow simulations: effect on pulse waves and parameter estimation. Commun. Comput. Phys. 4 (2008), 317-336. MR 2009h:76233

[2] D. Amadori, Initial-boundary value problems for nonlinear systems of conservation laws. NoDEA Nonlinear Differential Equations Appl. 4 (1997), 1-42. Zbl 0868.35069 MR 97m:35163

[3] D. Amadori and R. M. Colombo, Continuous dependence for $2 \times 2$ conservation laws with boundary. J. Differential Equations 138 (1997), 229-266. Zbl 0884.35091 MR 98g:35140

[4] S. S. Antman, Nonlinear problems of elasticity. 2nd ed., Appl. Math. Sci. 107, Springer, New York 2005. Zbl 1098.74001 MR 2006e:74001

[5] D. Armbruster, C. de Beer, M. Freitag, T. Jagalski, and C. Ringhofer, Autonomous control of production networks using a pheromone approach. Phys. A 363 (2006), 104-114. doi: 10.1016/j.physa.2006.01.052

[6] D. Armbruster, P. Degond, and C. Ringhofer, A model for the dynamics of large queuing networks and supply chains. SIAM J. Appl. Math. 66 (2006), 896-920. Zbl 1107.90002 MR 2006k:90015

[7] D. Armbruster, S. Göttlich, and M. Herty, A scalar conservation law with discontinuous flux for supply chains with finite buffers. SIAM J. Appl. Math. 71 (2011), 1070-1087. Zbl 1231.90154 MR 2823493

[8] A. Aw and M. Rascle, Resurrection of "second order" models of traffic flow. SIAM J. Appl. Math. 60 (2000), 916-938. Zbl 0957.35086 MR 2001a:35111

[9] M. K. Banda and M. Herty, Numerical discretization of stabilization problems with boundary controls for systems of hyperbolic conservation laws. Math. Control Relat. Fields $\mathbf{3}$ (2013), 121-142. Zbl 1262.65068 MR 3031137

[10] M. K. Banda, M. Herty, and A. Klar, Gas flow in pipeline networks. Netw. Heterog. Media 1 (2006), 41-56. Zbl 1108.76063 MR 2007d:76193

[11] M. K. Banda, M. Herty, and A. Klar, Coupling conditions for gas networks governed by the isothermal Euler equations. Netw. Heterog. Media 1 (2006), 295-314. Zbl 1109.76052 MR 2006m:35229

[12] M. K. Banda, M. Herty, and J.-M. T. Ngnotchouye, Coupling drift-flux models with unequal sonic speeds. Math. Comput. Appl. 15 (2010), 574-584. Zbl 1269.76091 MR 2012b:76149

[13] M. K. Banda, M. Herty, and J.-M. T. Ngnotchouye, Toward a mathematical analysis for drift-flux multiphase flow models in networks. SIAM J. Sci. Comput. 31 (2010), 4633-4653. Zbl 1213.35031 MR 2011f:35199

[14] G. Bastin, A. M. Bayen, C. D'Apice, X. Litrico, and B. Piccoli, Open problems and research perspectives for irrigation channels. Netw. Heterog. Media 4 (2009), i-v. MR 2525201

[15] G. Bastin, J.-M. Coron, and B. d'Andréa-Novel, Boundary feedback control and Lyapunov stability analysis for physical networks of $2 \times 2$ hyperbolic balance laws. In Proceedings of the 47th IEEE Conference on decision and control, Cancun, Mexico, 2008, 1454-1458. doi: 10.1109/CDC.2008.4738857 
[16] G. Bastin, J.-M. Coron, and B. d'Andréa-Novel, Using hyperbolic systems of balance laws for modeling, control and stability analysis of physical networks. found; see In Lecture notes for the Pre-congress workshop on complex embedded and networked control systems, 17th IFAC World Congress, Seoul, Korea 2008. http//:perso.uclouvain.be/georges.bastin/WS-Seoul-Bastin-et-al.pdf

[17] S. Bianchini, On the shift differentiability of the flow generated by a hyperbolic system of conservation laws. Discrete Contin. Dynam. Systems 6 (2000), 329-350. Zbl 1018.35051 MR 2000m:35120

[18] D. Blanchard and G. Griso, Asymptotic behavior of structures made of straight rods. $J$. Elasticity 108 (2012), 85-118. Zbl 06135624 MR 2922475

[19] S. Blandin, D. Work, P. Goatin, B. Piccoli, and A. Bayen, A general phase transition model for vehicular traffic. SIAM J. Appl. Math. 71 (2011), 107-127. Zbl 1217.35116 MR 2012b:35196

[20] R. Borsche, R. M. Colombo, and M. Garavello, On the coupling of systems of hyperbolic conservation laws with ordinary differential equations. Nonlinearity 23 (2010), 2749-2770. Zbl 1205.35170 MR 2011j:35154

[21] R. Borsche, R. M. Colombo, and M. Garavello, Mixed systems: ODEs - balance laws. J. Differential Equations 252 (2012), 2311-2338. Zbl 1252.35193 MR 2860620

[22] R. Borsche, R. Colombo, and M. Garavello, On the interactions between a solid body and a compressible inviscid fluid. Interfaces Free Bound. 15 (2013), 381-403. MR 3148597

[23] A. Bressan, Hyperbolic systems of conservation laws. Oxford Lecture Ser. Math. Appl. 20, Oxford University Press, Oxford 2000. Zbl 0997.35002 MR 2002d:35002

[24] A. Bressan, G. Crasta, and B. Piccoli, Well-posedness of the Cauchy problem for $n \times n$ systems of conservation laws. Mem. Amer. Math. Soc. 146 (2000), no. 694. Zbl 0958.35001 MR 2000m:35122

[25] A. Bressan and G. Guerra, Shift-differentiability of the flow generated by a conservation law. Discrete Contin. Dyn. Syst. 3 (1997), 35-58. Zbl 0948.35077 MR 97i:35108

[26] A. Bressan and K. Han, Optima and equilibria for a model of traffic flow. SIAM J. Math. Anal. 43 (2011), 2384-2417. Zbl 1236.90024 MR 2861667

[27] A. Bressan and K. Han, Nash equilibria for a model of traffic flow with several groups of drivers. ESAIM Control Optim. Calc. Var. 18 (2012), 969-986. Zbl 1262.35199 MR 3019468

[28] A. Bressan and K. Han, Existence of optima and equilibria for traffic flow on networks. Netw. Heterog. Media 8 (2013), 627-648. Zbl 1277.35248 MR 3117339

[29] A. Bressan, C. J. Liu, W. Shen, and F. Yu, Variational analysis of Nash equilibria for a model of traffic flow. Quart. Appl. Math. 70 (2012), 495-515. Zbl 1250.35173 MR 2986132

[30] A. Bressan, T.-P. Liu, and T. Yang, $L^{1}$ stability estimates for $n \times n$ conservation laws. Arch. Ration. Mech. Anal. 149 (1999), 1-22. Zbl 0938.35093 MR 2000g:35139

[31] A. Bressan and A. Marson, A maximum principle for optimally controlled systems of conservation laws. Rend. Sem. Mat. Univ. Padova 94 (1995), 79-94. Zbl 0935.49012 MR 97c:49025

[32] A. Bressan and W. Shen, Optimality conditions for solutions to hyperbolic balance laws. In Control methods in PDE-dynamical systems, Contemp. Math. 426, Amer. Math. Soc., Providence, RI, 2007, 129-152. Zbl 05194890 MR 2008b:49021 
[33] M. Bukač, S. Čanić, R. Glowinski, J. Tambača, and A. Quaini, Fluid-structure interaction in blood flow capturing non-zero longitudinal structure displacement. J. Comput. Phys. 235 (2013), 515-541. MR 3017610

[34] S. Čanić and E. H. Kim, Mathematical analysis of the quasilinear effects in a hyperbolic model blood flow through compliant axi-symmetric vessels. Math. Methods Appl. Sci. 26 (2003), 1161-1186. Zbl 1141.76484 MR 2004g:76139

[35] S. Čanić, A. Mikelić, D. Lamponi, and J. Tambača, Self-consistent effective equations modeling blood flow in medium-to-large compliant arteries. Multiscale Model. Simul. 3 (2005), 559-596. Zbl 1081.35073 MR 2005m:76207

[36] S. Čanić and B. Muha, Fluid-stent-artery interaction. In preparation.

[37] S. Čanić, B. Muha, and M. Bukač, Fluid-structure interaction in hemodynamics: Modeling, analysis, and numerical simulation. In Fluid-structure interaction in biomedical applications, Springer, to appear.

[38] S. Čanić and J. Tambača, Cardiovascular stents as PDE nets: 1D vs. 3D. IMA J. Appl. Math. (2012) 77, 748-770. Zbl 06128496 MR 2999136

[39] D. Q. Cao and R. W. Tucker, Nonlinear dynamics of elastic rods using the Cosserat theory: Modelling and simulation. Int. J. Solids Struct. 45 (2008), 460-477. Zbl 1167.74467 doi: 10.1016/j.ijsolstr.2007.08.016

[40] G. M. Coclite and M. Garavello, Vanishing viscosity for traffic on networks. SIAM J. Math. Anal. 42 (2010), 1761-1783. Zbl 1217.90053 MR 2011h:90031

[41] G. M. Coclite, M. Garavello, and B. Piccoli, Traffic flow on a road network. SIAM J. Math. Anal. 36 (2005), 1862-1886. Zbl 1114.90010 MR 2006e:90020

[42] R. M. Colombo and M. Garavello, On the Cauchy problem for the $p$-system at a junction. SIAM J. Math. Anal. 39 (2008), 1456-1471. Zbl 1155.35399 MR 2009a:35165

[43] R. M. Colombo, P. Goatin, and B. Piccoli, Road networks with phase transitions. $J$. Hyperbolic Differ. Equ. 7 (2010), 85-106. Zbl 1189.35176 MR 2011c:35329

[44] R. M. Colombo and G. Guerra, On general balance laws with boundary. J. Differential Equations 248 (2010), 1017-1043. Zbl 1196.35136 MR 2011b:35295

[45] R. M. Colombo, G. Guerra, M. Herty, and V. Schleper, Optimal control in networks of pipes and canals. SIAM J. Control Optim. 48 (2009), 2032-2050. Zbl 1196.35135 MR 2010e:49003

[46] R. M. Colombo, M. Herty, and V. Sachers, On $2 \times 2$ conservation laws at a junction. SIAM J. Math. Anal. 40 (2008), 605-622. Zbl 1171.35430 MR 2009m:35315

[47] R. M. Colombo and F. Marcellini, Coupling conditions for the $3 \times 3$ Euler system. Netw. Heterog. Media 5 (2010), 675-690. Zbl 06147577 MR 2011m:35219

[48] R. M. Colombo, F. Marcellini, and M. Rascle, A 2-phase traffic model based on a speed bound. SIAM J. Appl. Math. 70 (2010), 2652-2666. Zbl 1211.35183 MR 2011g:35240

[49] R. M. Colombo and C. Mauri, Euler system for compressible fluids at a junction. $J$. Hyperbolic Differ. Equ. 5 (2008), 547-568. Zbl 1168.35029 MR 2010b:35287

[50] J.-M. Coron, Control and nonlinearity. Math. Surveys Monogr. 136, Amer. Math. Soc., Providence, RI, 2007. Zbl 1140.93002 MR 2008d:93001 
[51] J.-M. Coron, B. d'Andréa Novel, and G. Bastin, A strict Lyapunov function for boundary control of hyperbolic systems of conservation laws. IEEE Trans. Automat. Control $\mathbf{5 2}$ (2007), 2-11. MR 2007k:93111

[52] R. Courant, K. Friedrichs, and H. Lewy, Über die partiellen Differenzengleichungen der mathematischen Physik. Math. Ann. 100 (1928), 32-74. JFM 54.0486.01

[53] E. Cristiani, C. de Fabritiis, and B. Piccoli, A fluid dynamic approach for traffic forecast from mobile sensor data. Commun. Appl. Ind. Math. 1 (2010), 54-71. MR 2812252

[54] J. A. Cunge, F. M. Holly, and A. Verwey, Practical aspects of computational river hydraulics. Pitmann, Boston 1980.

[55] C. M. Dafermos and L. Hsiao, Hyperbolic systems and balance laws with inhomogeneity and dissipation. Indiana Univ. Math. J. 31 (1982), 471-491. Zbl 0497.35058 MR 83m:35093

[56] C. D’Apice, S. Göttlich, M. Herty, and B. Piccoli, Modeling, simulation, and optimization of supply chains. Society for Industrial and Applied Mathematics (SIAM), Philadelphia, PA 2010. Zbl 1205.90003 MR 2011e:90001

[57] C. D’Apice and R. Manzo, A fluid dynamic model for supply chains. Netw. Heterog. Media 1 (2006), 379-398. Zbl 1131.90002 MR 2007f:35184

[58] C. D'Apice, R. Manzo, and B. Piccoli, Packet flow on telecommunication networks. SIAM J. Math. Anal. 38 (2006), 717-740. Zbl 1147.35331 MR 2007h:35221

[59] C. D'Apice, R. Manzo, and B. Piccoli, A fluid dynamic model for telecommunication networks with sources and destinations. SIAM J. Appl. Math. 68 (2008), 981-1003. Zbl 1149.35387 MR 2009c:35288

[60] C. D'Apice, R. Manzo, and B. Piccoli, Existence of solutions to Cauchy problems for a mixed continuum-discrete model for supply chains and networks. J. Math. Anal. Appl. 362 (2010), 374-386. Zbl 1180.35342 MR 2010k:35504

[61] M. de Saint-Venant, Théorie du mouvement non permanent des eaux, avec application aux crues des rivères et á l'introduction des marées dans leur lit. C. R. Hebd. Seanc. Acad. Sci. Paris 73 (1871), 147-154, 237-240. JFM 03.0482.04 http://gallica.bnf.fr/ark:/12148/bpt6k3030d/f147.image

[62] M. Dick, M. Gugat, and G. Leugering, Classical solutions and feedback stabilization for the gas flow in a sequence of pipes. Netw. Heterog. Media 5 (2010), 691-709. Zbl 1263.76059 MR 2012e:76108

[63] M. Dick, M. Gugat, and G. Leugering, A strict $H^{1}$-Lyapunov function and feedback stabilization for the isothermal Euler equations with friction. Numer. Algebra Control Optim. 1 (2011), 225-244. Zbl 1242.76296 MR 2012k:76072

[64] M. Á. Fernández, V. Milišić, and A. Quarteroni, Analysis of a geometrical multiscale blood flow model based on the coupling of ODEs and hyperbolic PDEs. Multiscale Model. Simul. 4 (2005), 215-236. Zbl 1085.35095 MR 2006c:76124

[65] L. Formaggia, F. Nobile, A. Quarteroni, and A. Veneziani, Multiscale modelling of the circulatory system: a preliminary analysis. Comput. Visual Sci. 2 (1999), 75-83. Zbl 1067.76624 doi: 10.1007/s007910050030

[66] L. Formaggia, A. Quarteroni, and A. Veneziani, Cardiovascular mathematics. MS\&A. Model. Simul. Appl. 1, Springer-Verlag Italia, Milano 2009. Zbl 05368508 MR 2010b:92034 
[67] L. Formaggia and A. Veneziani, Reduced and multiscale models for the human cardiovascular system. Notes of two lectures given by L. Formaggia at the 7th VKI Lecture Series on "Biological fluid dynamics" held at the Von Karman Institute, Belgium, in May 2003.

[68] T. L. Friesz, T. Kim, C. Kwon, and M. A. Rigdon, Approximate network loading and dual-time-scale dynamic user equilibrium. Transport. Res. B 45 (2011), 176-207. doi: 10.1016/j.trb.2010.05.003

[69] A. Fügenschuh, M. Herty, and A. Martin, Combinatorial and continuous models for the optimization of traffic flows on networks. SIAM J. Optim. 16 (2006), 1155-1176. Zbl 1131.90015 MR 2007k:90009

[70] M. Garavello and B. Piccoli, Traffic flow on a road network using the Aw-Rascle model. Comm. Partial Differential Equations 31 (2006), 243-275. Zbl 1090.90032 MR 2006k:90022

[71] M. Garavello and B. Piccoli, Traffic flow on networks. AIMS Ser. Appl. Math. 1, American Institute of Mathematical Sciences (AIMS), Springfield, MO, 2006. Conservation laws models. Zbl 1136.90012 MR 2008g:90023

[72] M. Garavello and B. Piccoli, Conservation laws on complex networks. Ann. Inst. H. Poincaré Anal. Non Linéaire 26 (2009), 1925-1951. Zbl 1181.35144 MR 2010j:35314

[73] M. Garavello and B. Piccoli, Coupling of Lighthill-Whitham-Richards and phase transition models. J. Hyperbolic Differ. Equ. 10 (2013), 577-636. Zbl 06222667 MR 3104083

[74] M. Garavello and B. Piccoli, Coupling of microscopic and phase transition models at boundary. Netw. Heterog. Media 8 (2013), 649-661. Zbl 1274.90076 MR 3117340

[75] S. K. Godunov, A difference method for numerical calculation of discontinuous solutions of the equations of hydrodynamics. Mat. Sb. (N.S.) 47 (89) (1959), 271-306 (in Russian). Zbl 0171.46204 MR 22 \#10194

[76] S. Göttlich, M. Herty, and A. Klar, Network models for supply chains. Commun. Math. Sci. 3 (2005), 545-559. Zbl 1115.90008 MR 2006f:90004

[77] S. Göttlich, M. Herty, and A. Klar, Modelling and optimization of supply chains on complex networks. Commun. Math. Sci. 4 (2006), 315-330. Zbl 1115.90009 MR 2006m:90073

[78] Q. Gu and T. Li, Exact boundary observability of unsteady flows in a tree-like network of open canals. Math. Methods Appl. Sci. 32 (2009), 395-418. Zbl 1155.35401 MR 2010b:93013

[79] M. Gugat, Nodal control of conservation laws on networks. In Control and boundary analysis, Lect. Notes Pure Appl. Math. 240, Chapman \& Hall/CRC, Boca Raton, FL, 2005, 201-215. Zbl 1097.65071 MR 2006b:49047

[80] M. Gugat, M. Herty, A. Klar, and G. Leugering, Optimal control for traffic flow networks. J. Optim. Theory Appl. 126 (2005), 589-616. Zbl 1079.49024 MR 2006m:49043

[81] M. Gugat, M. Herty, and V. Schleper, Flow control in gas networks: exact controllability to a given demand. Math. Methods Appl. Sci. 34 (2011), 745-757. Zbl 05883344 MR 2012j:93019

[82] M. Gugat and G. Leugering, Global boundary controllability of the de St. Venant equations between steady states. Ann. Inst. H. Poincaré Anal. Non Linéaire 20 (2003), 1-11. Zbl 1032.93030 MR 2003k:93007 
[83] J. C. Herrera, D. B. Work, R. Herring, X. Ban, Q. Jacobson, and A. M. Bayen, Evaluation of traffic data obtained via GPS-enabled mobile phones: The Mobile Century field experiment. Transport. Res. C 18 (2010), 568-583. doi: 10.1016/j.trc.2009.10.006

[84] M. Herty, Coupling conditions for networked systems of Euler equations. SIAM J. Sci. Comput. 30 (2008), 1596-1612. Zbl 1173.35080 MR 2009d:35217

[85] M. Herty, C. Kirchner, and A. Klar, Instantaneous control for traffic flow. Math. Methods Appl. Sci. 30 (2007), 153-169. Zbl 1117.90030 MR 2007k:93074

[86] M. Herty and A. Klar, Modeling, simulation, and optimization of traffic flow networks. SIAM J. Sci. Comput. 25 (2003), 1066-1087. Zbl 1046.90014 MR 2005f:90025

[87] M. Herty, A. Klar, and B. Piccoli, Existence of solutions for supply chain models based on partial differential equations. SIAM J. Math. Anal. 39 (2007), 160-173. Zbl 1165.90356 MR 2008m:49008

[88] M. Herty and M. Rascle, Coupling conditions for a class of second-order models for traffic flow. SIAM J. Math. Anal. 38 (2006), 595-616. Zbl 05139633 MR 2007e:35186

[89] M. Herty and C. Ringhofer, Averaged kinetic models for flows on unstructured networks. Kinet. Relat. Models 4 (2011), 1081-1096. Zbl 1242.90035 MR 2861587

[90] M. Herty and V. Sachers, Adjoint calculus for optimization of gas networks. Netw. Heterog. Media 2 (2007), 733-750. Zbl 1141.76057 MR 2008k:76106

[91] M. Herty and M. Seaïd, Simulation of transient gas flow at pipe-to-pipe intersections. Internat. J. Numer. Methods Fluids 56 (2008), 485-506. Zbl 1130.76068 MR 2382745

[92] M. Herty and M. Seaïd, Assessment of coupling conditions in water way intersections. Internat. J. Numer. Methods Fluids 71 (2013), 1438-1460. MR 3039443

[93] M. Hinze and K. Kunisch, Control strategies for fluid flows - optimal versus suboptimal control. In ENUMATH 97, World Scientific, Singapore 1997, 351-358. Zbl 1068.76512

[94] M. Hinze and K. Kunisch, On suboptimal control strategies for the Navier-Stokes equations. In Control and partial differential equations (Marseille-Luminy, 1997), ESAIM Proc. 4, Soc. Math. Appl. Indust., Paris 1998, 181-198.Zbl 0912.49019 MR 2000i:49031

[95] H. Holden and N. H. Risebro, A mathematical model of traffic flow on a network of unidirectional roads. SIAM J. Math. Anal. 26 (1995), 999-1017. Zbl 0833.35089 MR 96h:90046

[96] H. Holden and N. H. Risebro, Riemann problems with a kink. SIAM J. Math. Anal. 30 (1999), 497-515. Zbl 0926.35087 MR 2000b:35161

[97] D. Kröner, Numerical schemes for conservation laws. Wiley-Teubner Series Advances in Numerical Mathematics, John Wiley \& Sons Ltd., Chichester; Teubner, Stuttgart 1997. Zbl 0872.76001 MR 98b:65003

[98] L. D. Landau and E. M. Lifshitz, Course of theoretical physics. Vol. 6, 2nd ed., Pergamon Press, Oxford 1987. Zbl 04070476 MR 89i:00006

[99] C. Lattanzio and B. Piccoli, Coupling of microscopic and macroscopic traffic models at boundaries. Math. Models Methods Appl. Sci. 20 (2010), 2349-2370. Zbl 1215.35103 MR 2012c:35267

[100] J.-P. Lebacque, Les modèles macroscopiques du traffic. Ann. Ponts Chaussées 67 (1993), $24-45$. 
[101] J.-P. Lebacque and M. Khoshyaran, First order macroscopic traffic flow models for networks in the context of dynamic assignment. In Transportation Planning, Appl. Optim. 64, Kluwer Academic Publishers, New York 2002, 119-140. Zbl 1050.90507 doi: 10.1007/0-306-48220-7_8

[102] G. Leugering and J. P. G. Schmidt, On the modelling and stabilization of flows in networks of open canals. SIAM J. Control Optim. 41 (2002), 164-180. Zbl 1024.76009 MR 2003k:76033

[103] G. Leugering and J. Sokolowski, Topological derivatives for networks of elastic strings. ZAMM Z. Angew. Math. Mech. 91 (2011), 926-943. Zbl 05989482 MR 2854546

[104] R. J. LeVeque, Finite volume methods for hyperbolic problems. Cambridge Texts Appl. Math., Cambridge University Press, Cambridge 2002. Zbl 1010.65040 MR 2003h:65001

[105] T. Li, Exact controllability for quasilinear hyperbolic systems and its application to unsteady flows in a network of open canals. Math. Methods Appl. Sci. 27 (2004), 1089-1114. Zbl 1151.93316 MR 2005b:76030

[106] T. Li, Exact boundary controllability of unsteady flows in a network of open canals. Math. Nachr. 278 (2005), 278-289. Zbl 1066.93005 MR 2005h:93090

[107] T. Li and B. Rao, Contrôlabilité exacte frontière de l'écoulement d'un fluide nonstationnaire dans un réseau du type d'arbre de canaux ouverts. C. R. Math. Acad. Sci. Paris 339 (2004), 867-872. Zbl 1116.93311 MR 2005h:93018

[108] T. Li and Z. Wang, Global exact boundary controllability for first order quasilinear hyperbolic systems of diagonal form. Int. J. Dyn. Syst. Differ. Equ. 1 (2007), 12-19. Zbl 1168.35388 MR 2010b:93018

[109] M. J. Lighthill and G. B. Whitham, On kinematic waves. II. A theory of traffic flow on long crowded roads. Proc. Roy. Soc. London. Ser. A. 229 (1955), 317-345. Zbl 0064.20906 MR 17,310a

[110] E. S. Menon, Gas pipeline hydraulics. CRC Press, Boca Raton, FL, 2005.

[111] A. Mikelić and S. Čanić, Homogenization closure for a two-dimensional effective model describing fluid-structure interaction in blood flow. In Math everywhere, Springer-Verlag, Berlin 2007, 193-205. Zbl 05223213 MR 2008a:74036

[112] T. Nishida, Global solution for an initial boundary value problem of a quasilinear hyperbolic system. Proc. Japan Acad. 44 (1968), 642-646. Zbl 0167.10301 MR 38 \#4821

[113] T. Nishida and J. Smoller, Mixed problems for nonlinear conservation laws. J. Differential Equations 23 (1977), 244-269. Zbl 0303.35052 MR 55 \#882

[114] M. Olufsen, C. S. Peskin, W. Y. Kim, E. M. Pedersen, A. Nadim, and L. Jesper, Numerical simulation and experimental validation of blood flow in arteries with structured-tree outflow conditions. Ann. Biomed. Eng. 28 (2000), 1281-1299.

[115] G. Parke and N. Hewson, ICE manual of bridge engineering. 2nd ed., ICE Publishing, London 2008.

[116] A. Quarteroni, Cardiovascular mathematics. In Proc. Internat. Congress of Mathematicians. Vol. I, Eur. Math. Soc., Zürich 2007, 479-512. Zbl 1121.92022 MR 2008f:92021

[117] P. I. Richards, Shock waves on the highway. Operations Res. 4 (1956), 42-51. MR 17,761b 
[118] M. Sablé-Tougeron, Méthode de Glimm et problème mixte. Ann. Inst. H. Poincaré Anal. Non Linéaire 10 (1993), 423-443. Zbl 0832.35093 MR 94k:35192

[119] M. C. Steinbach, On PDE solution in transient optimization of gas networks. J. Comput. Appl. Math. 203 (2007), 345-361. Zbl 1119.93045 MR 2008h:90141

[120] D. Sun, I. S. Strub, and A. M. Bayen, Comparison of the performance of four Eulerian network flow models for strategic air traffic management. Netw. Heterog. Media 2 (2007), 569-595. Zbl 1143.90006 MR 2009e:90008

[121] J. Tambača, M. Kosor, S. Čanić, and D. Paniagua, Mathematical modeling of endovascular stents. SIAM J. Appl. Math. 70 (2010), 1922-1952. Zbl 05837575 MR 2011f:74048

[122] S. Ulbrich, Optimal control of nonlinear hyperbolic conservation laws with source terms. Habilitation thesis, Technical University München, München 2001.

[123] D. B. Work, S. Blandin, O.-P. Tossavainen, B. Piccoli, and A. M. Bayen, A traffic model for velocity data assimilation. Appl. Math. Res. Express. AMRX (2010), 1-35. Zbl 1187.90098 MR 2773421

[124] H. M. Zhang, A non-equilibrium traffic model devoid of gas-like behavior. Transport. Res. B 36 (2002), 275-290. doi: 10.1016/S0191-2615(00)00050-3

[125] P. Zunino, Drug-eluting stents modeled using 1d hyperbolic network. Private communication.

Received December 6, 2013; revised January 20, 2014 